\title{
Adipogenic placenta-derived mesenchymal stem cells are not lineage restricted by withdrawing extrinsic factors: developing a novel visual angle in stem cell biology
}

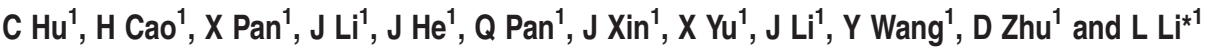

Current evidence implies that differentiated bone marrow mesenchymal stem cells (BMMSCs) can act as progenitor cells and transdifferentiate across lineage boundaries. However, whether this unrestricted lineage has specificities depending on the stem cell type is unknown. Placental-derived mesenchymal stem cells (PDMSCs), an easily accessible and less invasive source, are extremely useful materials in current stem cell therapies. No studies have comprehensively analyzed the transition in morphology, surface antigens, metabolism and multilineage potency of differentiated PDMSCs after their dedifferentiation. In this study, we showed that after withdrawing extrinsic factors, adipogenic PDMSCs reverted to a primitive cell population and retained stem cell characteristics. The mitochondrial network during differentiation and dedifferentiation may serve as a marker of absent or acquired pluripotency in various stem cell models. The new population proliferated faster than unmanipulated PDMSCs and could be differentiated into adipocytes, osteocytes and hepatocytes. The cell adhesion molecules (CAMs) signaling pathway and extracellular matrix (ECM) components modulate cell behavior and enable the cells to proliferate or differentiate during the differentiation, dedifferentiation and redifferentiation processes in our study. These observations indicate that the dedifferentiated PDMSCs are distinguishable from the original PDMSCs and may serve as a novel source in stem cell biology and cell-based therapeutic strategies. Furthermore, whether PDMSCs differentiated into other lineages can be dedifferentiated to a primitive cell population needs to be investigated.

Cell Death and Disease (2016) 7, e2141; doi:10.1038/cddis.2016.1; published online 17 March 2016

Stem-cell-based therapies have gradually become a hot topic due to their high plasticity and self-renewing ability; clinical investigations with stem cell products in regenerative medicine are addressing a wide spectrum of conditions using a variety of stem cell types. These pluripotent cells including embryonic stem cells (ESCs), termed induced pluripotent stem cells (iPSCs), were first tested but inhibited in their clinical applications owing to ethical and tumorigenic problems. As a promising candidate for tissue regeneration, mesenchymal stem cells (MSCs) are fibroblast-like, with high plasticity and self-renewing ability and are able to develop into diverse cell lineages. ${ }^{1}$ Among the MSCs from different adult tissues, placental-derived mesenchymal stem cells (PDMSCs), which reside in the fetal membranes of the term placenta, are easily accessible and less invasive. Their abundance, high proliferative potency, short population doubling time, strong immunosuppression and lack of ethical concerns make them indispensable in stem cell research and therapy. ${ }^{2}$

Specific growth factors, cytokines and extracellular matrix components may have an important role in the determination of stem cell fate by switching from self-renewal to a differentiation stage. During lineage alteration to a specific tissue cell type, it was thought that MSCs progressively and developmentally became lineage restricted. ${ }^{3}$ Yet some evidences have suggested that when terminally differentiated mammalian cells are cultured under special conditions, they will revert to a more primitive phenotype. ${ }^{4-6}$ More recently, in the presence of human embryonic stem cell medium supplemented with valproic acid, stem cells derived from amniotic fluid could be fully reprogrammed to pluripotency

\footnotetext{
${ }^{1}$ Collaborative Innovation Center for Diagnosis and Treatment of Infectious Diseases, State Key Laboratory for Diagnosis and Treatment of Infectious Diseases, School of Medicine, First Affiliated Hospital, Zhejiang University, Hangzhou, China

${ }^{*}$ Corresponding author: L Li, Collaborative Innovation Center for Diagnosis and Treatment of Infectious Diseases, State Key Laboratory for Diagnosis and Treatment of Infectious Diseases, School of Medicine, First Affiliated Hospital, Zhejiang University, Hangzhou 571, China. Tel: +86 57187236759 ; Fax: +86 571 87236755 ; E-mail: ljil @zju.edu.cn

Abbreviations: ESCs, embryonic stem cells; iPSCs, induced pluripotent stem cells; MSCs, mesenchymal stem cells; PDMSCs, placental-derived mesenchymal stem cells; MET, mesenchymal-to-epithelial transition; BMMSCs, bone marrow mesenchymal stem cells; OXPHOS, oxidative phosphorylation; CAMs, cell adhesion molecules; ECM, extracellular matrix; DePDMSCs, dedifferentiated PDMSCs; NNT, nicotinamide nucleotide transhydrogenase; NADPH, nicotinamide adenine dinucleotide phosphate; AL, adipocyte-like; PPARG, peroxisome proliferator-activated receptor-g; ATP5A1, ATP synthase, H+ transporters, mitochondrial F1 complex, alpha subunit 1; COX4I1, cytochrome $c$ oxidase subunit IV isoform 1; MT-CO1, mitochondrially encoded cytochrome $c$ oxidase I; MT-CO2, mitochondrially encoded cytochrome $c$ oxidase II; TFAM, transcription factor A, mitochondrial; TOMM34, translocase of outer mitochondrial membrane 34; LONP1, lon peptidase 1, mitochondrial; PPAR-a, peroxisome proliferator-activated receptor alpha; RUNX2, runt-related transcription factor 2; OC, osteocalcin; CYP, cytochrome P; ALB, albumin; BSA, bovine serum albumin; PE, phycoerythrin; FITC, fluorescein isothiocyanate; AFP, alpha-fetoprotein; CK18, cytokeratin 18; CK19, cytokeratin 19; $\mathrm{H}_{2} \mathrm{O}_{2}$, hydrogen peroxide; PAS, periodic acid-schiff staining; ICG, indocyanine green; FGF7, fibroblast growth factor 7; ZNF711, zinc finger protein 711; MMP10, matrix metallopeptidase 10; CADM1, cell adhesion molecule 1 Received 11.8.15; revised 26.11.15; accepted 18.12.15; Edited by Y Shi
} 
a

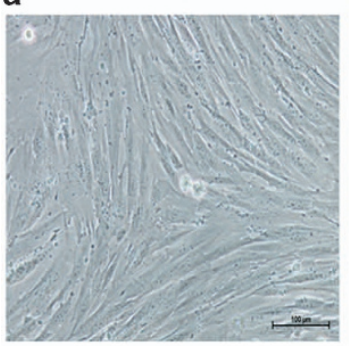

d

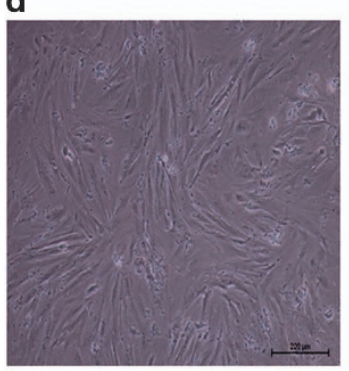

b

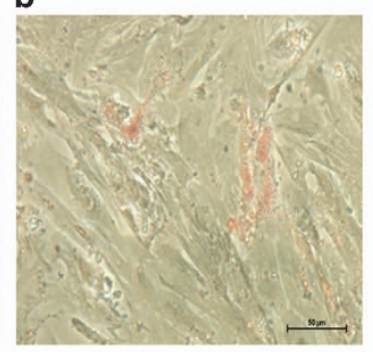

e

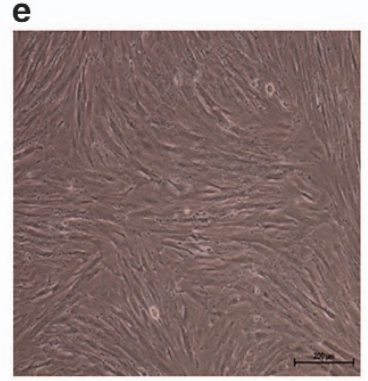

C

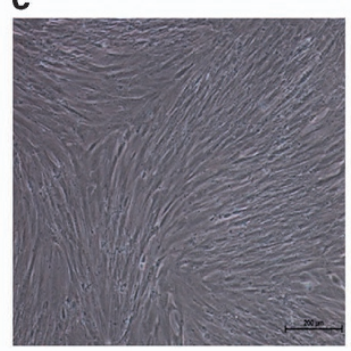

f

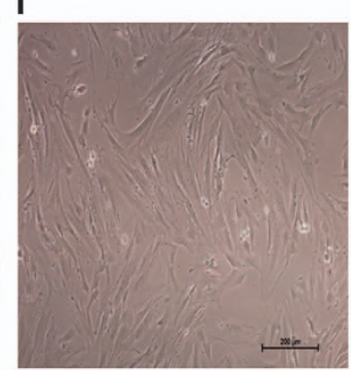

g
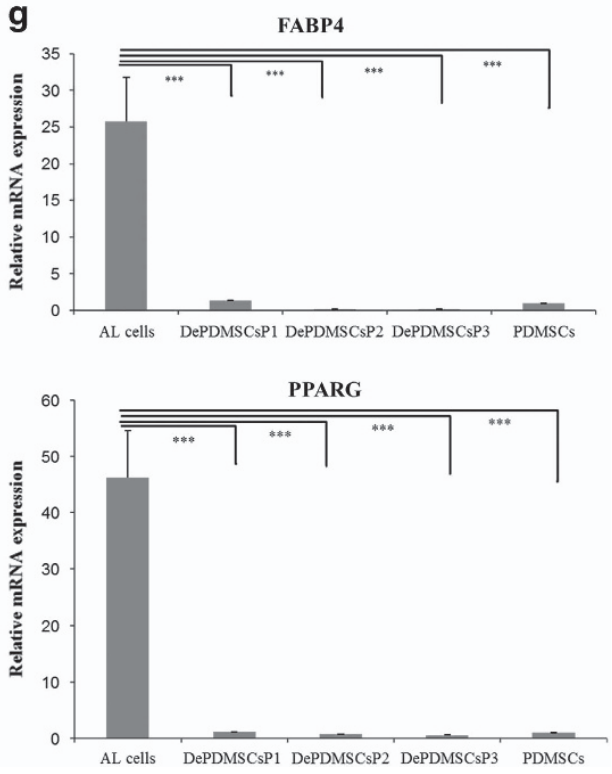

Figure 1 Morphology and adipocyte markers of adipogenic PDMSCs reverted to a primitive state after dedifferentiation. (a) Morphology of PDMSC P3 showed a long spindle shape. Scale bar, $100 \mu \mathrm{m}$. (b) AL cells after Oil Red O staining demonstrated lipid accumulation. Scale bar, $50 \mu \mathrm{m}$. (c) The nonstimulated controls were negative for Oil Red $\mathrm{O}$ staining. Scale bar, $200 \mu \mathrm{m}$. (d) DePDMSCs in general PDMSCs medium at day 2. Scale bar, $100 \mu \mathrm{m}$. (e) DePDMSCs in general PDMSCs medium at day 7. Scale bar, $100 \mu \mathrm{m}$. (f) DePDMSCs at passage 3 occupied a similar shape as PDMSCs P3 in culture. Scale bar, $100 \mu \mathrm{m}$. (g) RT-QPCR analysis of FABP4 and PPARG in PDMSCs, AL cells and DePDMSCs from passages 1 to 3 . The expression of the PDMSC group was normalized to 1 . Data are presented as the mean \pm S.D. in triplicate and were statistically analyzed by one-way ANOVA ( $n=3$ independent donor cells); ${ }^{* *} P<0.001$

without genetic manipulation. ${ }^{7}$ This process was defined as dedifferentiation and is considered as one of the mechanisms to reroute cell fate. ${ }^{8}$ Furthermore, a downregulation of lineagespecific genes and an upregulation of stem genes occurred immediately after initiation of the dedifferentiation process. ${ }^{8}$ This phase was characterized by repression of somatic genes via methylation, increased cell proliferation, altered morphology, signal transduction changes, reactivation of telomerase activity and the mesenchymal-to-epithelial transition (MET). ${ }^{9,10}$ MET includes the loss of mesenchymal characteristics, such as motility, and the acquisition of epithelial characteristics such as cell polarity and the expression of cell adhesion molecules. ${ }^{11}$

In addition, bone marrow mesenchymal stem cells (BMMSCs) which were induced into osteocytes, chondrocytes and adipocytes, can dedifferentiate into a primitive population on the withdrawal of stimulating culture medium. ${ }^{12-14}$ This new population correlated with cell cycle arrest and associated genes, had enhanced cell survival, greater efficacy in differentiation and improved therapeutic potential in vitro and in vivo compared with uncommitted BMMSCs. ${ }^{15,16}$ On the other hand, a number of studies showed enhanced mitochondrial biogenesis in various stem cell differentiation models including ESCs and iPSCs. ${ }^{17,18}$ The immature mitochondrial phenotype in ESCs consists of fewer mitochondria, poorly developed cristae and a perinuclear location of mitochondria. ${ }^{19,20}$ These characteristics have been regarded as potential markers of pluripotency in ESCs; ${ }^{20}$ however, it has not been clearly established whether the morphology and the mitochondrial network is pluripotency dependent or stem cell specific. In addition, it has been suggested that mitochondrial dynamics and oxidative phosphorylation (OXPHOS) activity can influence each other during the biological process. ${ }^{21}$ Consequently, we suggest that the altered OXPHOS activity will accompany the differentiation and dedifferentiation processes.

In the present study, we aimed to comprehensively analyze the transition in morphology, surface antigens, metabolism and multilineage potency during PDMSCs differentiation and dedifferentiation to clarify whether unrestricted lineage exists in differentiated PDMSCs. We showed that after withdrawing extrinsic factors, adipogenic PDMSCs reverted to a primitive cell population and retained stem cell characteristics. The new population proliferated faster than unmanipulated PDMSCs, and could be differentiated into adipocytes, osteocytes and hepatocytes. Gene expression profiling showed a panel of genes with significantly up- or downregulated expression between adipogenically differentiated and dedifferentiated cells. The cell adhesion molecules (CAMs) signaling pathway and extracellular matrix (ECM) components modulate cell behavior and enable the cells to regenerate, proliferate and differentiate during the differentiation, dedifferentiation and redifferentiation processes. ${ }^{22,23}$ These observations indicated that the dedifferentiated PDMSCs were distinguishable from the original PDMSCs and may serve as a novel source in stem cell biology and cell-based therapeutic strategies.

\section{Results}

Morphology and adipocyte markers of adipogenic PDMSCs reverted to a primitive state after dedifferentiation. PDMSCs at passage 3 displayed a long spindle shape (Figure 1a). After adipogenesis for 14 days, PDMSCs 
a
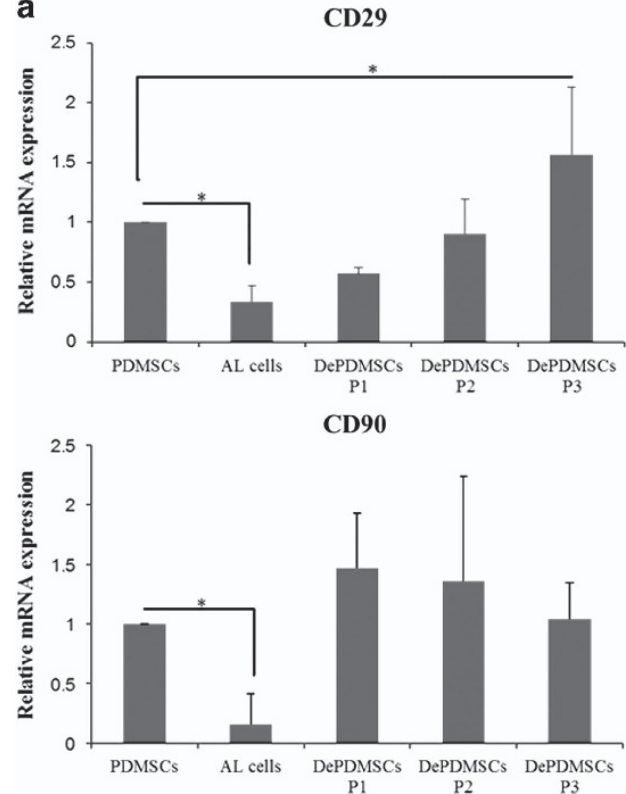

b
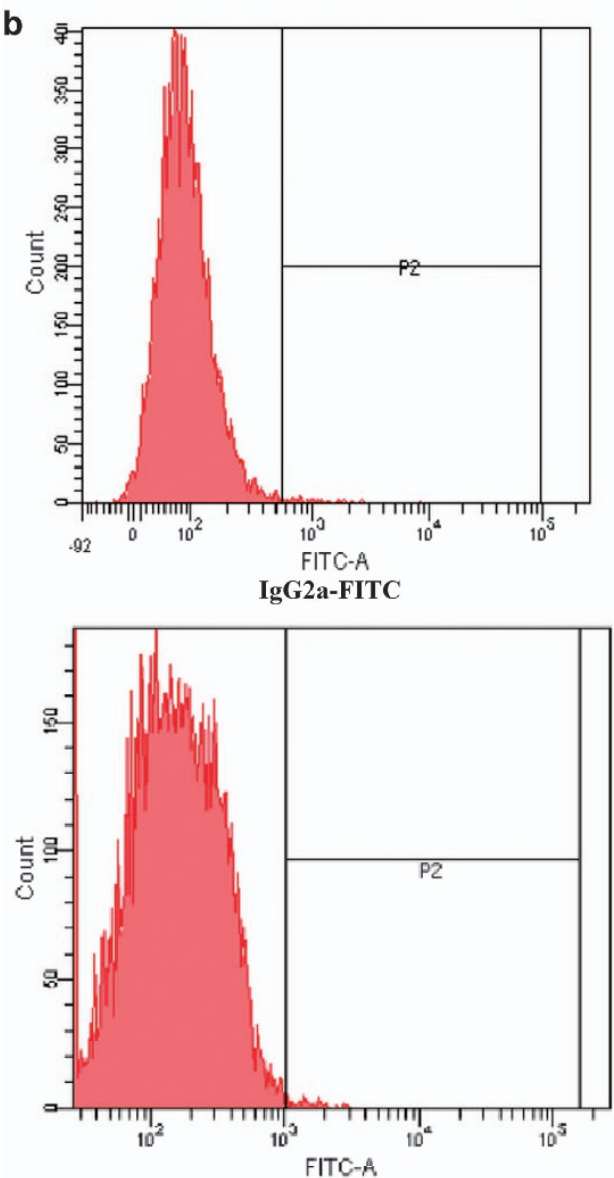

IgG1-FITC

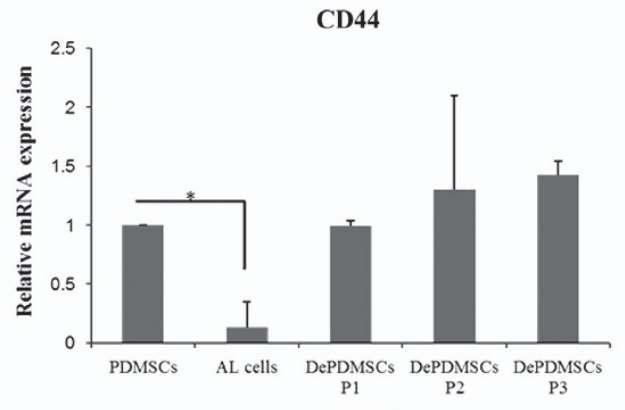

CD105
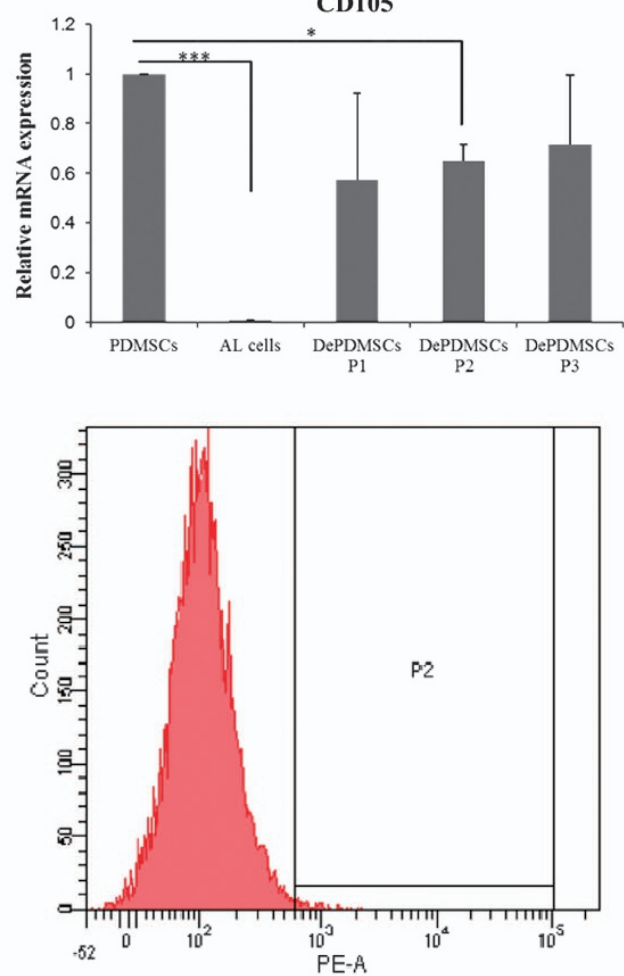

IgG2a-PE/Cy5.5 ${ }^{\circledR}$

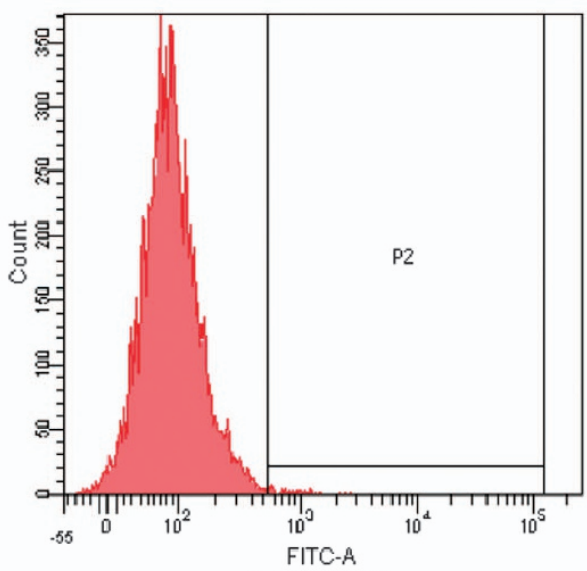

IgG2b-FITC

Figure 2 Positive surface antigens and ESC markers expression of DePDMSCs reverted to a primitive state, but the negative markers were not altered all the time. (a) RT-QPCR analysis of the expression of CD29, CD44, CD90 and CD105 on PDMSCs P3, AL cells and DePDMSCs from passages 1 to 3. (b) Immunophenotype of the negative controls determined by flow cytometry using labeled antibodies. (c) Immunophenotype of PDMSCs at passage 3, AL cells and DePDMSCs at passage 3 determined by flow cytometry using labeled antibodies specific for the indicated human surface antigens. (d) FACS analysis of PDMSCs at passage 3 , AL cells and DePDMSCs at passage 3. Data are presented as the mean \pm S.D. in triplicate and were statistically analyzed by one-way ANOVA ( $n=3$ independent donor cells); ${ }^{*} P<0.05,{ }^{* \star} P<0.01$. (e) CD34 and the embryonic stem cell markers expression of PDMSCs and DePDMSCs determined by immunocytochemistry; scale bars, $100 \mu \mathrm{m}$ 
exhibited the round shape characteristics of cultured adipocyte (Figure 1b) and thus were termed as adipocyte-like (AL) cells. AL cells showed an initiation of lipid droplet formation as shown by positive Oil Red $\mathrm{O}$ staining (Figure 1b). The nonstimulated controls were negative for Oil Red $O$ staining (Figure 1c). However, withdrawal of adipogenic medium c
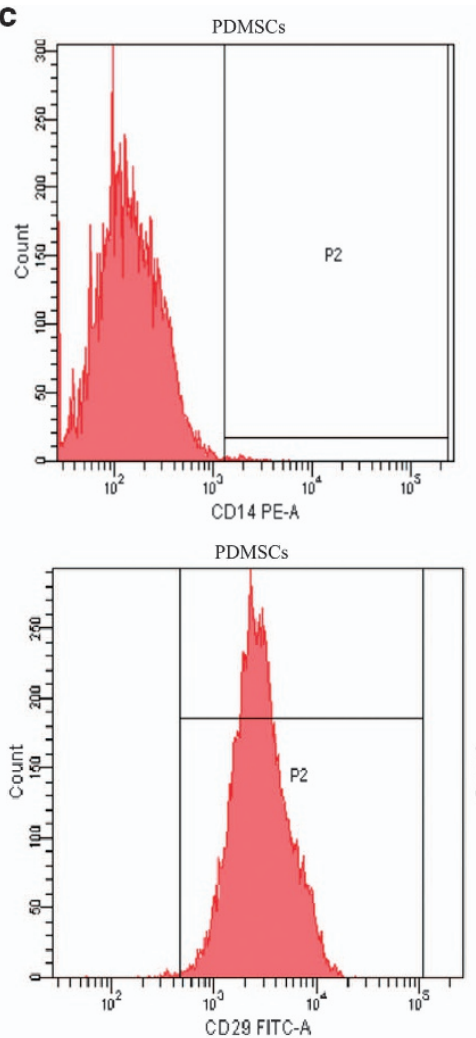

PDMSCs
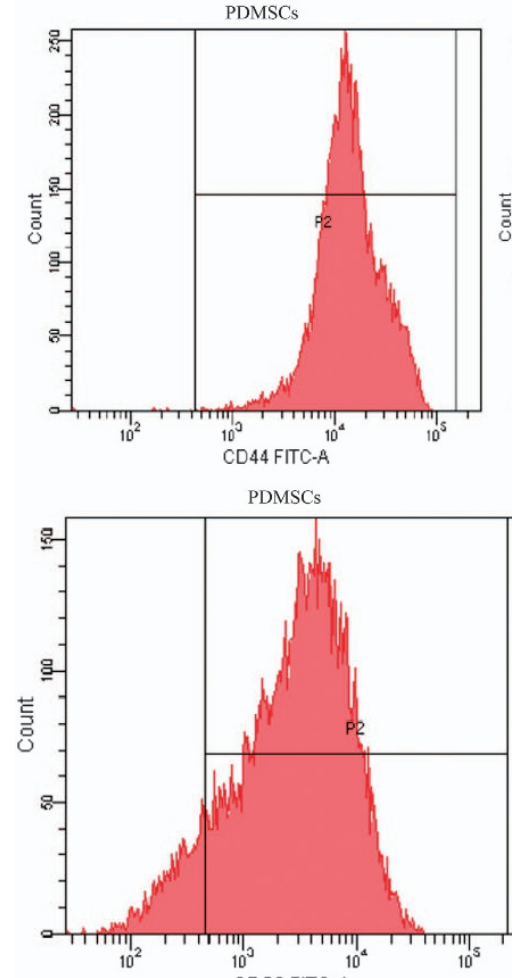

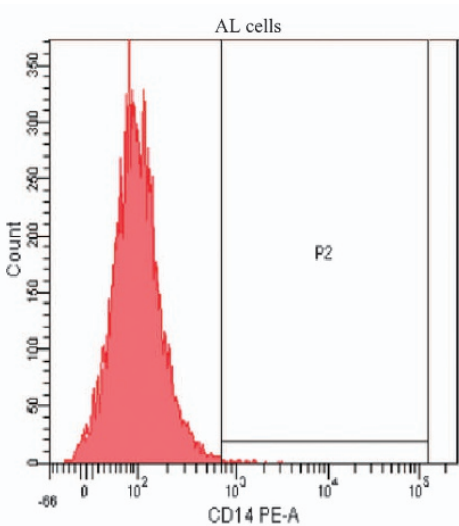

AL cells

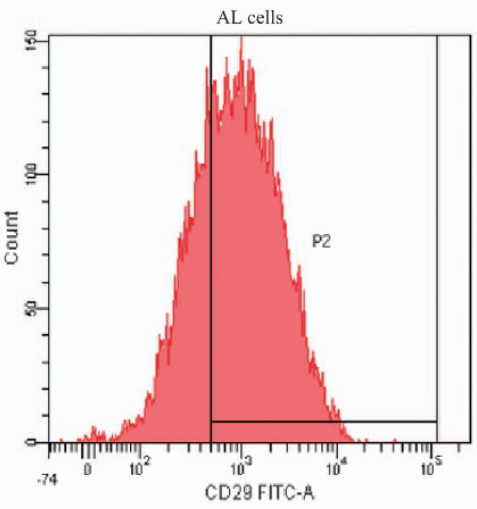

AL cells
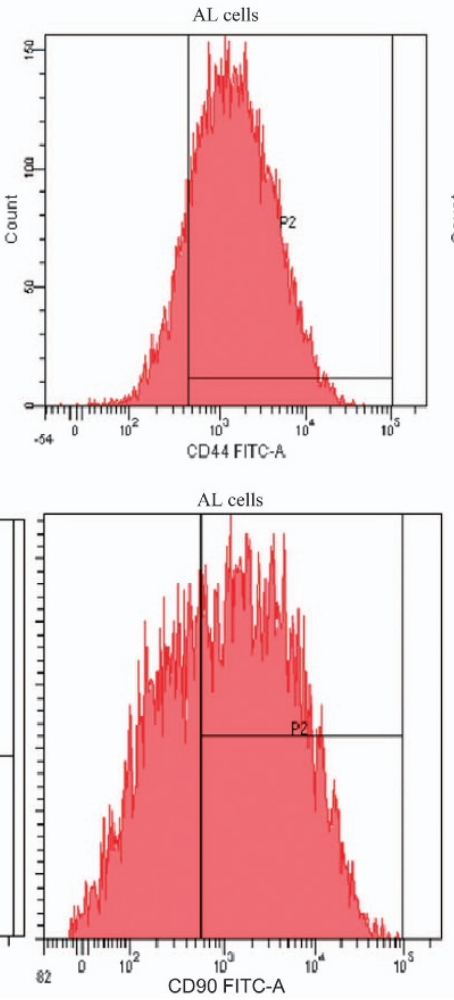

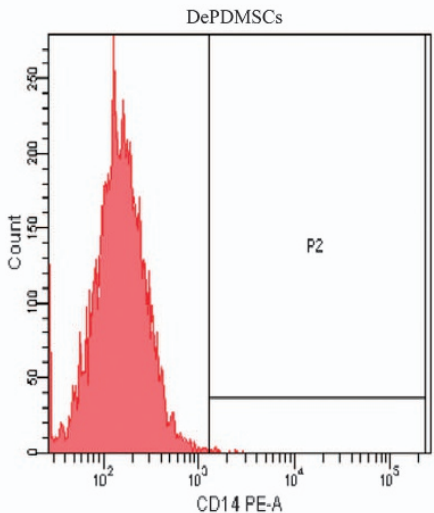

DePDMSCs
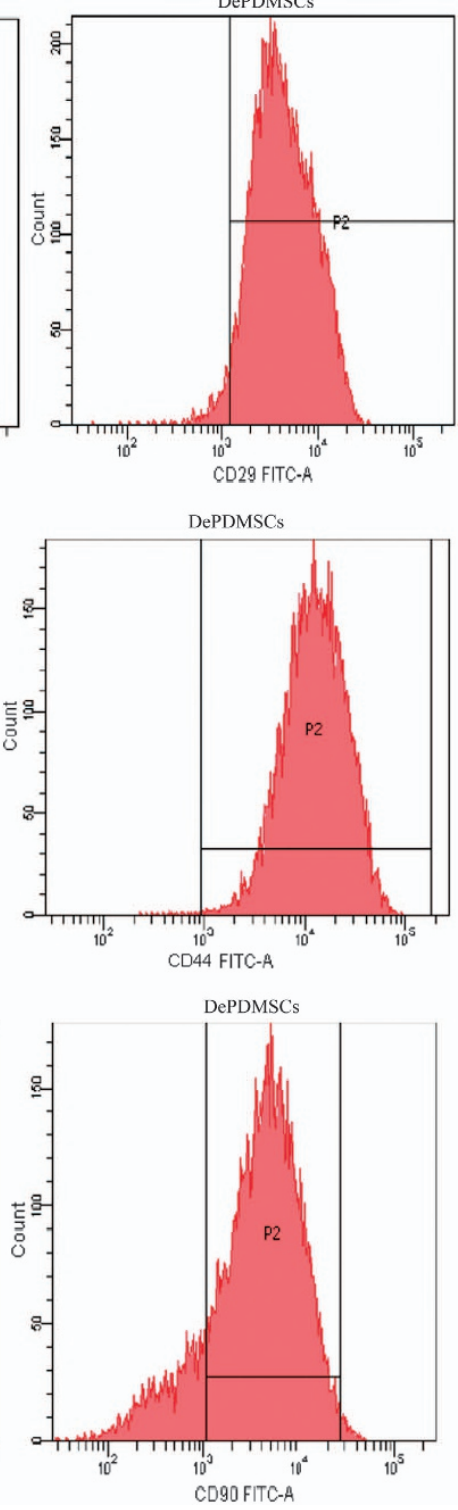

Figure 2 Continued 

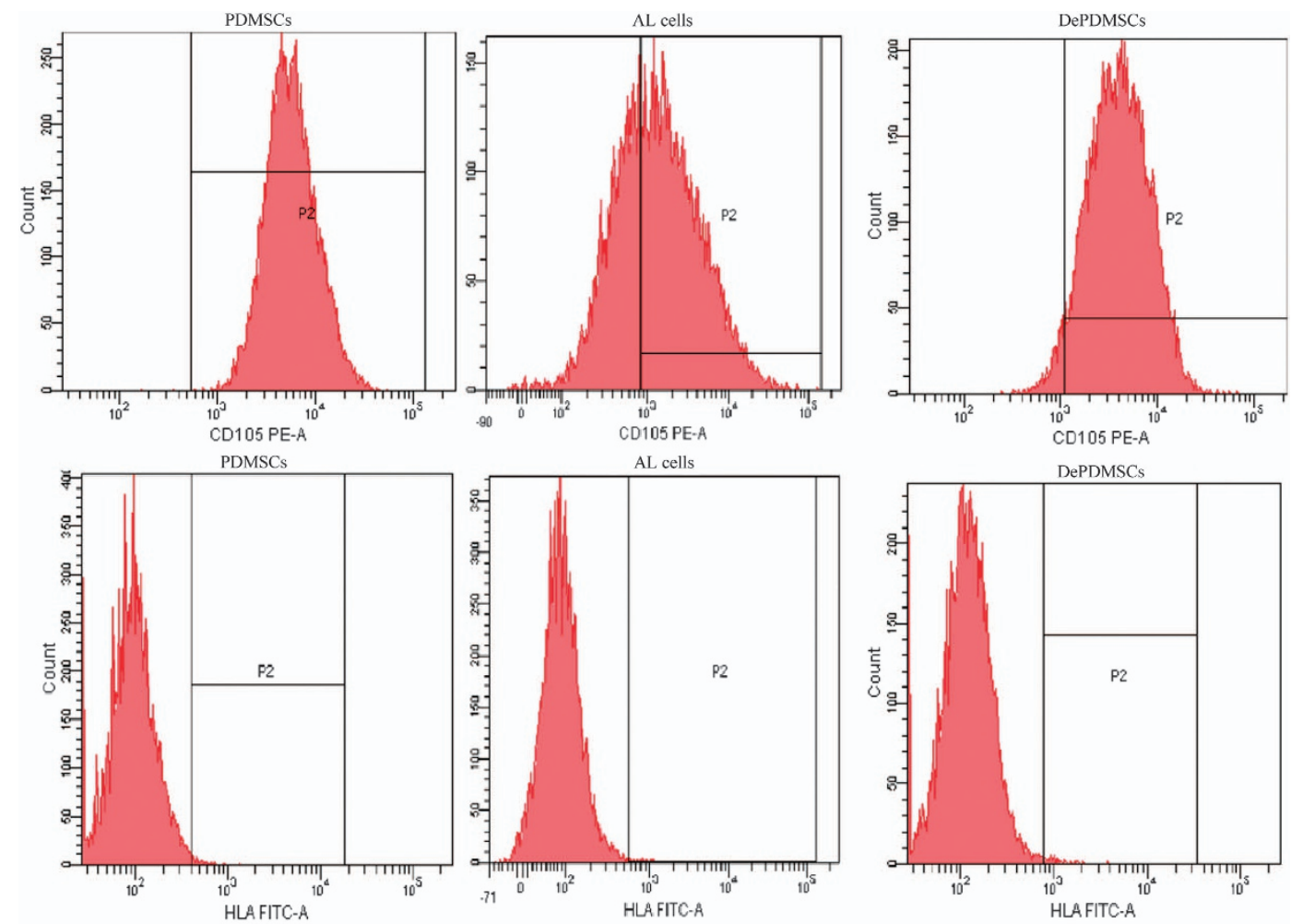

d

Flow cytometry

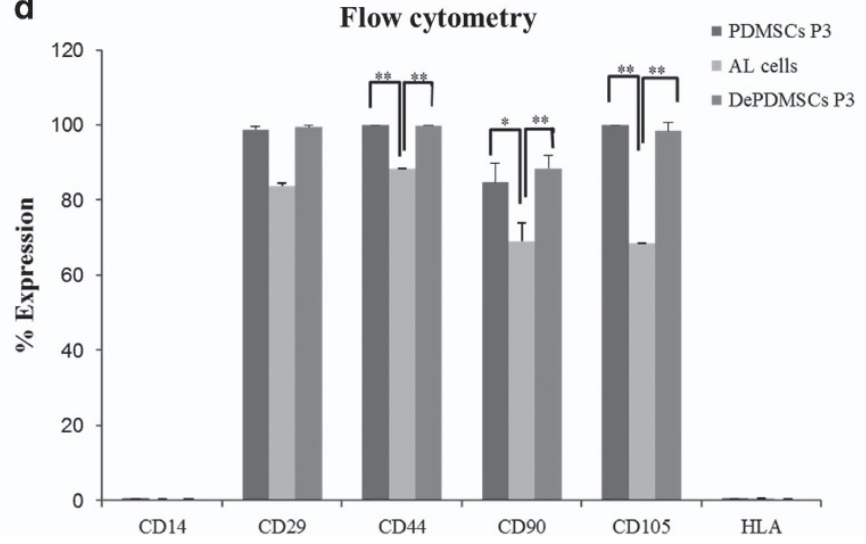

Figure 2 Continued

rapidly reverted $A L$ cells to the long spindle morphology from day 2 to day 7 (Figures $1 \mathrm{~d}$ and e). Then, the dedifferentiated PDMSCs was passaged (Figure 1f) and cultured in general PDMSC medium for another 14 days. At the molecular level, the expression of the adipocyte-specific genes FABP4 and PPARG were significantly upregulated at day 14 of adipogenesis when compared with undifferentiated PDMSCs $(P<0.001)$. After withdrawing adipogenic medium, the markers were significantly downregulated to a similar level as PDMSCs, and there was no difference between each passage of dedifferentiated PDMSCs (Figure 1g).

Surface antigen expression of adipogenic PDMSCs at the mRNA and protein levels reverted to a primitive state after dedifferentiation. As previously reported, PDMSCs positively express mesenchymal markers such as CD29, CD44, CD90 and CD105, but negatively express the hematopoietic markers CD14 and HLA-DR. ${ }^{24}$ The expression levels in PDMSCs were set to 1 for normalization. Positive markers for CD29, CD44, CD90 and CD105 mRNA expression levels were significantly decreased in $A L$ cells and then increased after dedifferentiation in general PDMSC medium (Figure 2a). The CD29 mRNA level in DePDMSCs at passage 3 was significantly higher than the level in PDMSCs at passage $3(P<0.05)$. The CD105 mRNA level in AL cells was significantly downregulated compared with PDMSCs at passage $3(P<0.001)$, and the mRNA expression level in DePDMSCs at passage 2 was lower than PDMSCs at passage $3(P<0.05)$. However, the expression returned to a level comparable to PDMSCs at passage 3 after culture in 

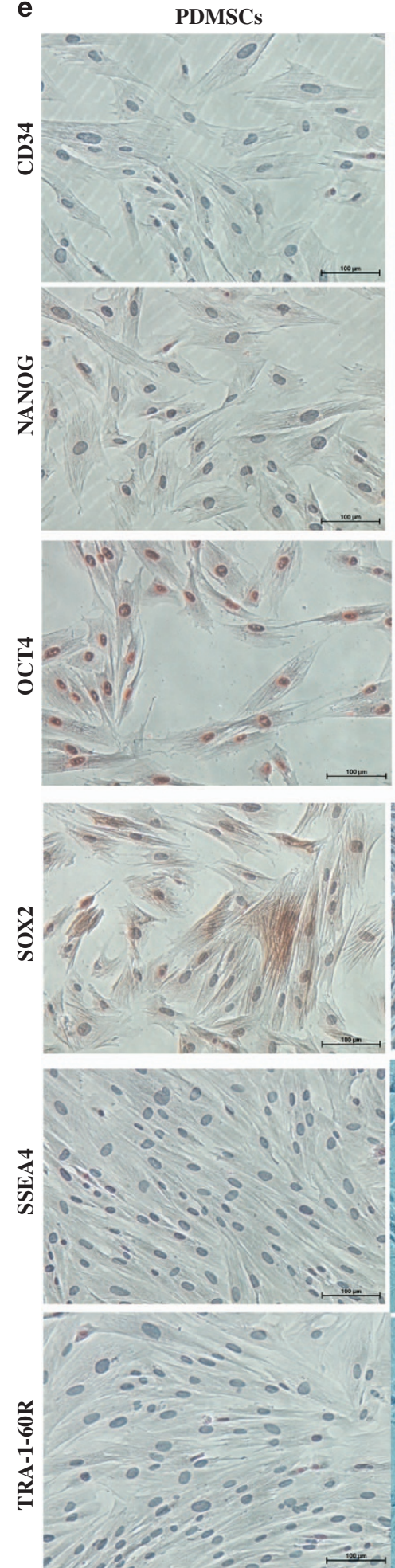

AL cells
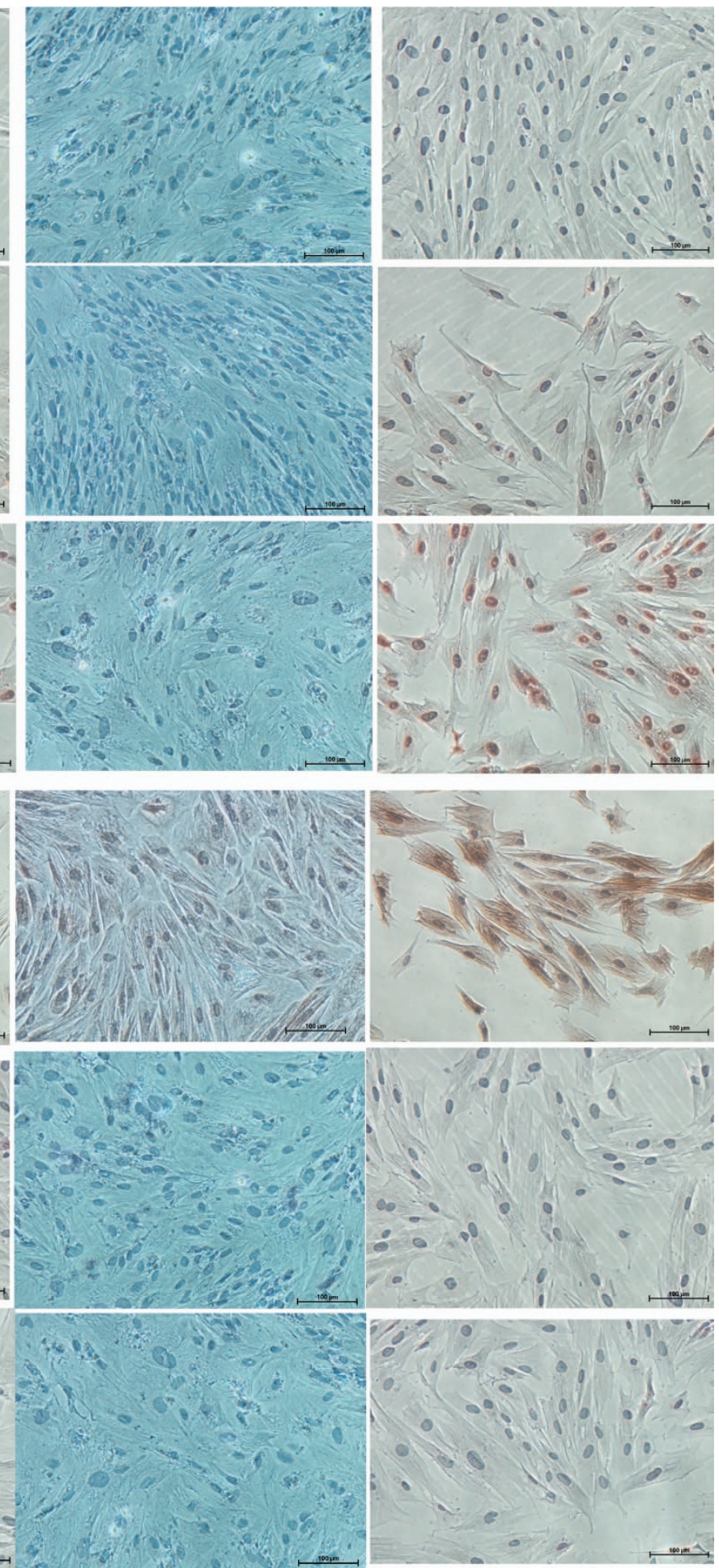

DePDMSCs
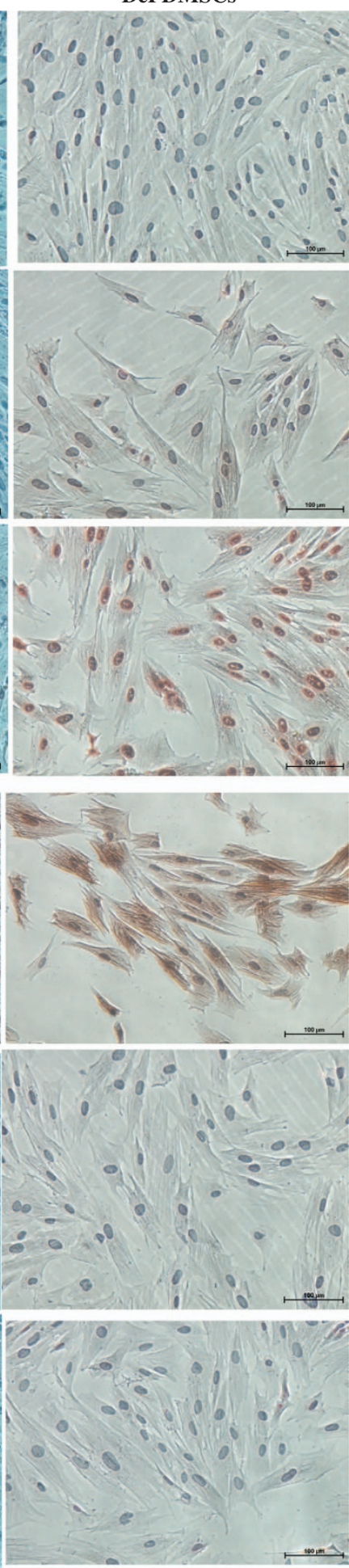

Figure 2 Continued

general PDMSC medium for 21 days; cells at this stage were termed dedifferentiated PDMSCs (DePDMSCs) at passage 3 and were used for the subsequent analysis.

The immunophenotype of the negative controls was determined by flow cytometry using labeled antibodies
(Figure 2b). The immunophenotype of PDMSCs, AL cells and DePDMSCs at passage 3 was determined by flow cytometry (Figure 2c). PDMSCs showed positive expression for CD29, CD44, CD90 and CD105, but negative expression for CD14 and HLA-DR. After adipogenic differentiation for 
a

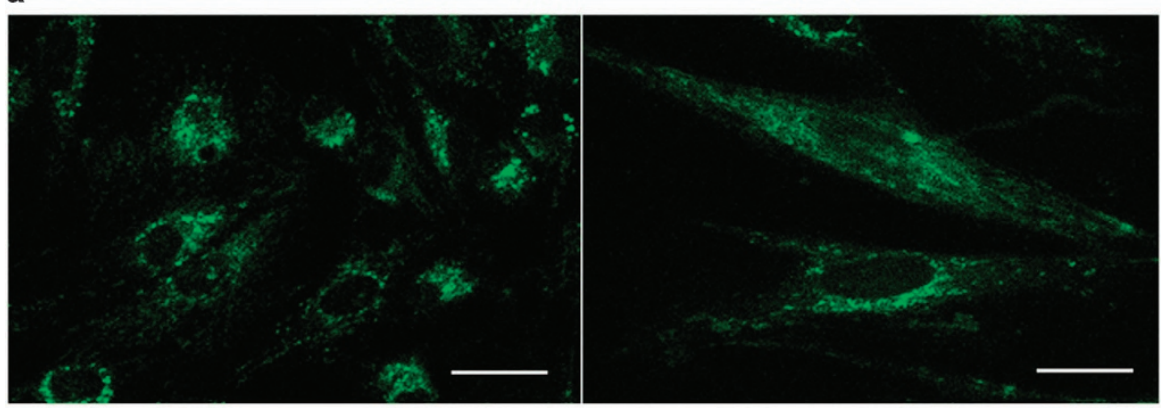

b

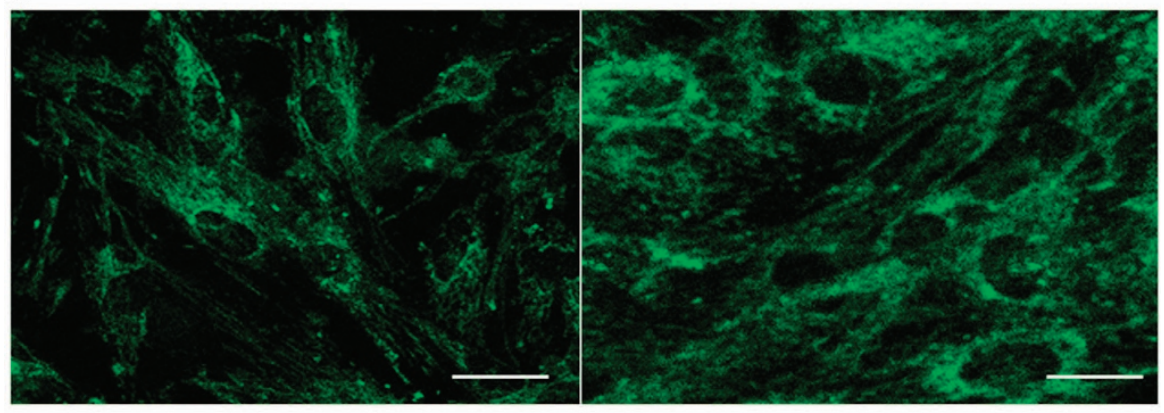

C

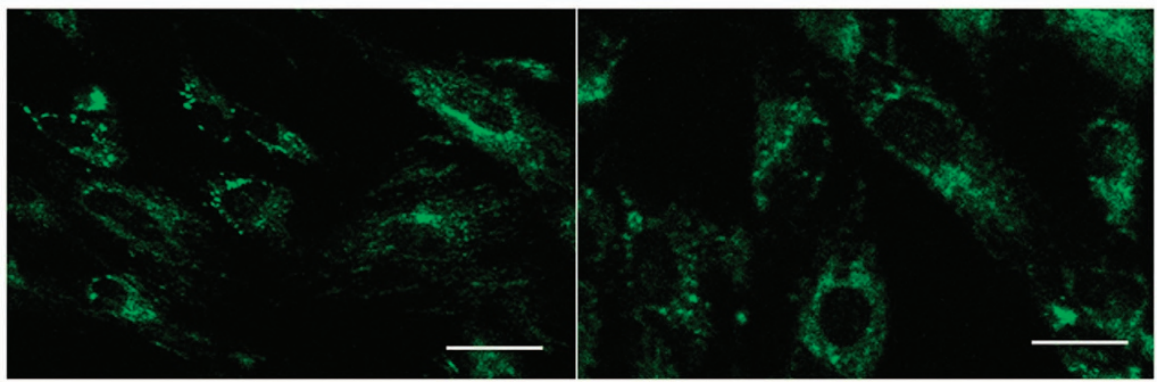

Figure 3 The mitochondrial network of PDMSCs underwent changes during adipogenic differentiation and dedifferentiation. Representative bright-field micrographs of (a) PDMSCs at passage 3, (b) AL cells and (c) DePDMSCs at passage 3 stained with MitoTracker Green FM (green); left, scale bars, $100 \mu \mathrm{m}$; right, scale bars, $50 \mu \mathrm{m}$. (d) RT-QPCR demonstrated increased mRNA expression of several mitochondrial biogenesis regulators after the adipogenic differentiation of PDMSCs, and these specific genes were downregulated after dedifferentiation. The expression levels of these genes in PDMSCs were normalized to 1. (e) OXPHOS related proteins expression of PDMSCs, AL cells and DePDMSCs were determined by the OXPHOS Magnetic Bead Panel. Data are presented as the mean \pm S.D. in triplicate and were statistically analyzed by one-way ANOVA ( $n=3$ independent donor cells); ${ }^{*} P<0.05,{ }^{\star \star} P<0.01,{ }^{\star \star \star} P<0.001$

14 days, $A L$ cells showed relatively lower expression for the positive markers in comparison with PDMSCs at passage 3; the negative makers were not altered. Then, after dedifferentiation for 21 days, DePDMSCs at passage 3 had a comparable expression pattern of positive and negative surface antigens to PDMSCs at passage 3 . The results in our study showed that CD44, CD90 and CD105 were significantly altered during adipogenic differentiated PDMSCs $(P<0.05)$ but reverted to a comparable level after dedifferentiation; the hematopoietic markers were not altered throughout the differentiation and dedifferentiation processes (Figure 2d).

Immunocytochemistry ( $n=3$; Figure $2 \mathrm{e})$ of the PDMSCs at passage 3 revealed highly positive expression for SOX2, weakly positive expression for OCT4 and negative expression for CD34, NANOG, SSEA4 and TRA-1-60R. After adipogenic differentiation for 14 days, $A L$ cells showed downregulated expression for the positive embryonic stem cell markers
(OCT4 and SOX2), while they returned to a level similar to uncommitted PDMSCs after dedifferentiation for 21 days. Conversely, the negative markers were not altered during the adipogenic differentiation and dedifferentiation processes.

\section{The mitochondrial network of PDMSCs undergoes} changes during adipogenic differentiation and dedifferentiation. Although the globular shape and perinuclear localization of mitochondria in ESCs and iPSCs has been regarded as a potential marker of pluripotency, ${ }^{20}$ it has not been clearly established whether the morphology of the mitochondrial network is pluripotency dependent or stem cell specific. Staining of the mitochondrial network revealed that expanding PDMSCs display a developed network composed of thread-like mitochondria spread throughout the cytoplasm (Figure 3a); adipogenic differentiation resulted in cells with more and larger round-shaped mitochondria (Figure $3 b$ ). After dedifferentiation, DePDMSCs also displayed a 

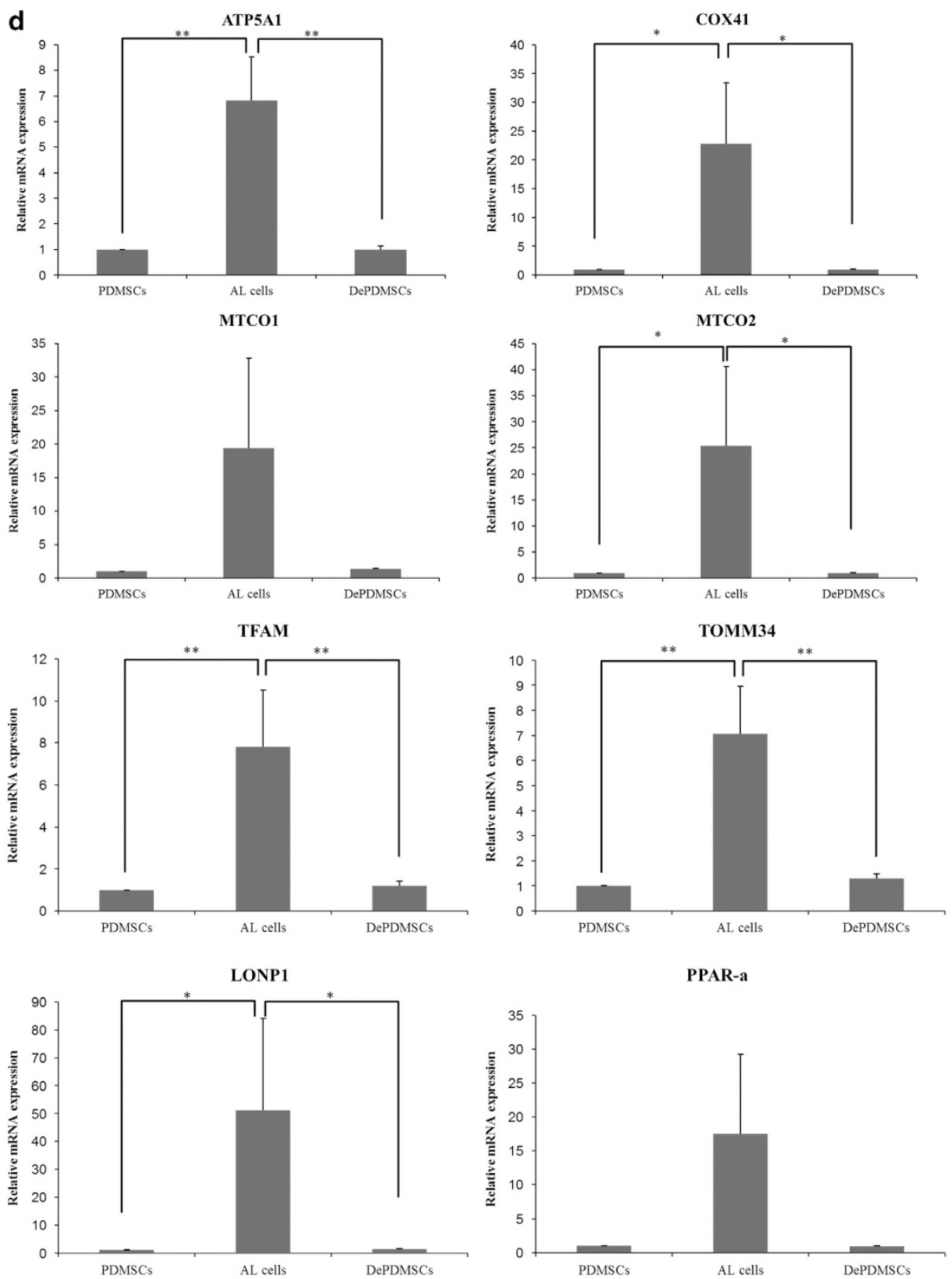

PPAR-a

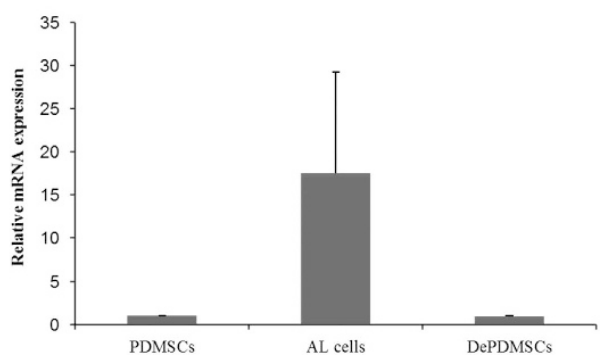

Figure 3 Continued

mitochondrial population similar to PDMSCs with a simultaneously decreased quantity (Figure $3 c$ ).

Given that mitochondrial biogenesis also requires the synthesis and import of many mitochondrial proteins, we next analyzed the abundance of several mitochondria specific genes at the mRNA level throughout the processes. These regulators included ATP5A1, COX4I1, MT-CO1, MT-CO2, TFAM, TOMM34, LONP1 and PPAR-a. We showed a trend towards mRNA abundance of all mitochondria specific genes in $A L$ cells when compared with PDMSCs at passage 3 , but they were downregulated in DePDMSCs at passage 3 (Figure 3d). ATP5A1, TFAM and TOMM34 in AL cells showed a significantly increased level compared with undifferentiated PDMSCs or DePDMSCs $(P<0.01)$. COX4I1, MT-CO2 and
LONP1 in AL cells also showed a significantly increased level compared with undifferentiated PDMSCs or DePDMSCs $(P<0.05)$. However, MT-CO1 and PPAR-a demonstrated a higher trend but there was no difference in $A L$ cells when compared with PDMSCs and DePDMSCs. These data further support the notion that an enhanced or decreased mitochondrial biogenesis process occurs during the differentiation and dedifferentiation processes.

OXPHOS is the main source of energy in eukaryotic cells. The results of a Human OXPHOS Magnetic Bead Panel (Figure $3 e$ ) showed a significantly increased trend for the nicotinamide nucleotide transhydrogenase (NNT) protein during the adipogenic process, but it decreased to the original level after dedifferentiation $(P<0.01)$. Complex I was 
e
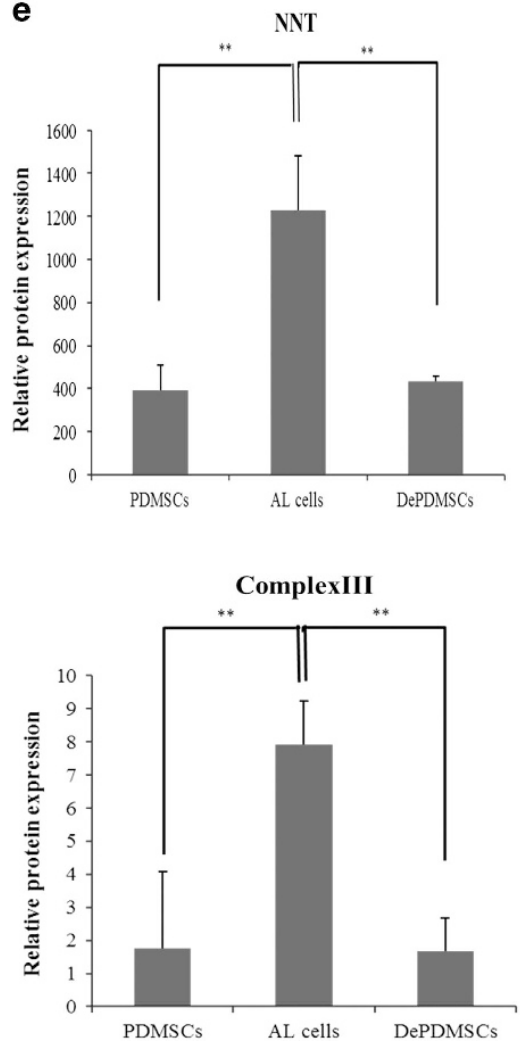
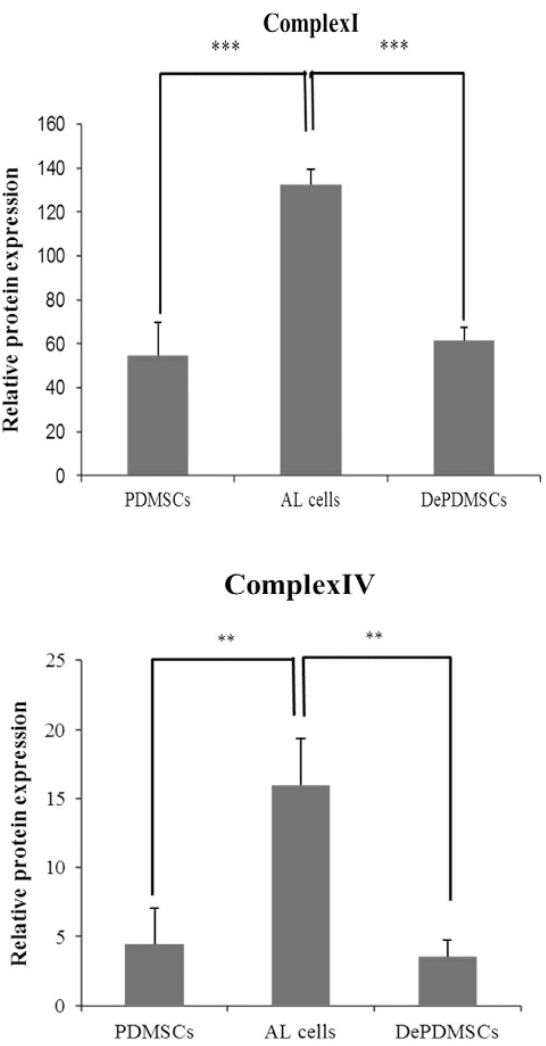

ComplexII
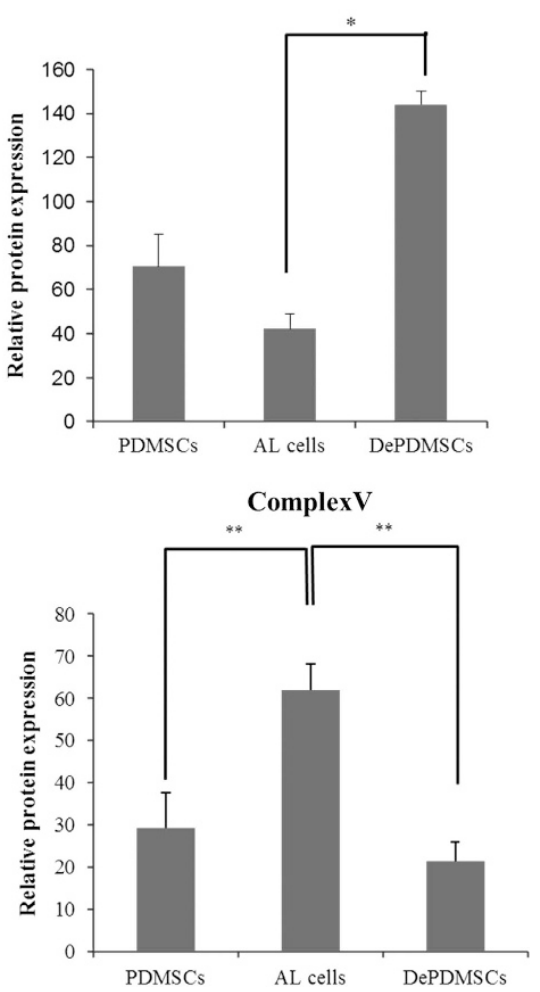

Figure 3 Continued

upregulated in $\mathrm{AL}$ cells when compared with PDMSCs but downregulated in DePDMSCs $(P<0.001)$; Complex III, Complex IV and Complex V demonstrated similar alterations during the differentiation and dedifferentiation processes $(P<0.01)$. Although the complex II protein level was lower in $A L$ cells when compared with DePDMSCs at passage $3(P<0.05)$, there was no difference between AL cells and PDMSCs. With the exception of NNT, the other OXPHOS proteins were generally at a low level during the differentiation and dedifferentiation processes.

DePDMSCs can grow more quickly than uncommitted PDMSCs and expanding PDMSCs. We further detected the proliferation ability of DePDMSCs and compared this with uncommitted PDMSCs and expanding PDMSCs. We found that the proliferation rates (Figure 4a) of PDMSCs at passage 3 , PDMSCs at passage 6 and DePDMSCs at passage 3 were slow during the first 2-3 days (latent phase) and then accelerated rapidly during 4-6 days (logarithmic phase) and thereafter slowed down (stationary phase). The doubling time of PDMSCs at passage 3 in the logarithmic phase was $2.16 \pm 0.16 \mathrm{~d}$, PDMSCs at passage 6 was $3.46 \pm 0.25 \mathrm{~d}$ and DePDMSCs at passage 3 was $1.65 \pm 0.26 d$ (Figure $4 b$ ). The proliferation ability results showed that DePDMSCs at passage 3 grew more quickly than PDMSCs at passage 3 $(P<0.05)$. The proliferation ability of DePDMSCs at passage 3 and PDMSCs at passage 3 was higher than PDMSCs at passage $6(P<0.001)$.
The multilineage differentiation ability of DePDMSCs was comparable to PDMSCs. To clarify whether the new dedifferentiated population was able to obtain multilineage differentiation ability, we further induced the new population via adipogenic, osteogenic and hepatogenic differentiation and compared them with PDMSCs.

After PDMSCs and DePDMSCs were induced in adipogenic medium for 14 days, they acquired the typical characteristics of adipocytes, and Oil red $O$ staining showed lipid accumulation (Figures $5 \mathrm{a}$ and $\mathrm{d}$ ). DePDMSCs retained adipogenic potency and the mRNA expression levels of FABP4 and PPARG in adipogenic DePDMSCs were higher than adipogenic PDMSCs. Human adipose tissue was used as a positive control (Figure 5e). Immunocytochemistry of FABP4 and PPARG showed negative expression in PDMSCs and DePDMSCs at day 0 , and strongly positive expression at day 14 of adipogenesis (Figure 5f).

After PDMSCs and DePDMSCs were induced in osteogenic medium for 14 days, they acquired the typical characteristics of osteocytes, and alizarin red staining showed calcium accumulation (Figures $6 a$ and $d$ ). DePDMSCs retained osteogenic potency and the mRNA expression levels of RUNX2 and osteocalcin in osteogenic DePDMSCs were comparable to osteogenic PDMSCs. Osteoblasts were used as a positive control (Figure 6e). Immunocytochemistry of RUNX2 and osteocalcin showed negative expression in PDMSCs and DePDMSCs at day 0, and strongly positive expression at day 14 of osteogenesis (Figure 6f). 


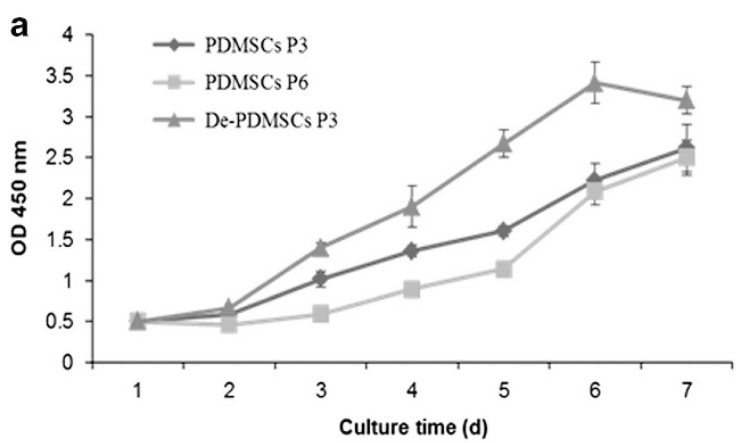

b

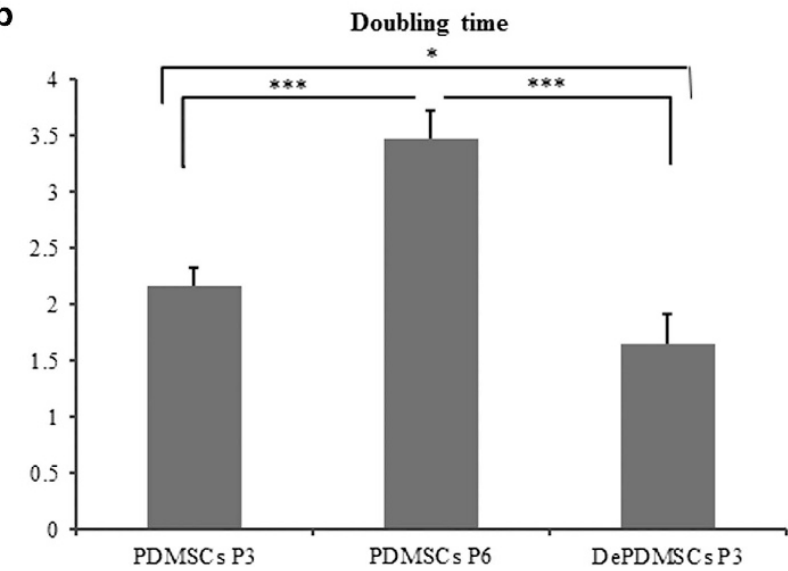

Figure 4 DePDMSCs can grow more quickly than unmanipulated PDMSCs and expanding PDMSCs. The growth curves of PDMSCs P3, PDMSCs P6 and DePDMSCs P3 were detected by a Cell Proliferation Reagent WST-1 assay at $450 \mathrm{~nm}$ on a spectrophotometer $(n=3)$. (a) The proliferation rates of PDMSCs at passage 3, PDMSCs at passage 6 and DePDMSCs at passage 3. (b) The doubling time of PDMSCs at passage 3 , PDMSCs at passage 6 and DePDMSCs at passage 3 . Data are presented as the mean \pm S.D. in triplicate and were statistically analyzed by one-way ANOVA ( $n=3$ independent donor cells); ${ }^{*} P<0.05$, ${ }^{* * *} P<0.001$

After PDMSCs and DePDMSCs were induced stepwise in hepatogenic medium for 21 days, the long spindle cells gradually turned to the classical cubic morphology of hepatocytes (Figures 7a and f). RT-QPCR analysis revealed significantly upregulated mRNA expression of ALB, CYP1A2 and CYP3A4 in PDMSCs and DePDMSCs at day 21 of hepatogenic differentiation. Human liver tissue was used as a positive control (Figure 7g). Immunocytochemistry (Figures $7 \mathrm{~h}$ and i) revealed the presence of hepatic markers (AFP, CK18 and CK19) in hepatogenic PDMSCs and DePDMSCs that was weakly positive at day 8 and of moderate intensity at day 21 . Hepatogenic PDMSCs and DePDMSCs could also uptake and release ICG; however, the expanding PDMSCs and DePDMSCs could not uptake ICG (Figure 7j). Periodic acidSchiff staining (PAS) staining showed that both PDMSCs and DePDMSCs could store more glycogen after hepatogenic induction for 21d (Figure 7k).

Numerous genes and multiple signaling pathways cooperate to regulate the adipogenic differentiation and dedifferentiation processes. Gene expression profiling was performed to obtain a deeper molecular insight into $\mathrm{AL}$ cells and their subsequent dedifferentiation to primitive cell types with multilineage potency. GeneChips were generated for PDMSCs, AL cells and DePDMSCs from three donors. We selected 2140 out of 49395 probe sets that represented genes with differential expression between AL cells and PDMSCs after removing double entries and probe sets with no title (Supplementary Table S1). Among them, the expression levels of 952 genes were upregulated and 1188 were downregulated on day 14 of adipogenesis. Using the same criteria, we selected 2486 out of 49395 probe sets that represented genes with differential expression between $A L$ cells and DePDMSCs (Supplementary Table S2). Among them, the expression levels of 1590 genes were upregulated and 896 were downregulated on day 21 of dedifferentiation. On the basis of KEGG pathway enrichment analysis, the upregulated and downregulated differentially expressed genes were mainly enriched in the multiple crucial KEGG pathways listed in Tables $1 a$ and $b$. We then identified nine differentially expressed genes in DePDMSCs compared with PDMSCs (Supplementary Table S3), all genes except for fibroblast growth factor 7 (FGF7) were upregulated in DePDMSCs when compared with PDMSCs. KEGG pathway enrichment analysis demonstrated that three of the differentially expressed genes were involved in multiple pathways (Table 1c). To confirm the microarray analysis results, RTQPCR analysis (Supplementary Figure S1) showed that the expression levels of most genes in DePDMSCs were consistent with the microarray data except for FGF7. Herein, the expression of cell adhesion molecule 1 (CADM1) was upregulated in DePDMSCs more than sevenfold when compared with PDMSCs $(P<0.001)$. Meanwhile, the expression of matrix metallopeptidase 10 (MMP10) in DePDMSCs was upregulated for more than sevenfold $(P<0.01)$, the expression of zinc finger protein 711 (ZNF711) in DePDMSCs was upregulated for more than threefold $(P<0.05)$. Among them, CADM1 was included in the CAMs signaling pathway and could be beneficial to identify the central nodes within the signaling web of ECM on functional basis. $^{25}$

\section{Discussion}

In contrast to the lineage restriction in differentiated cells, it has been reported that cell fate is interconvertible. ${ }^{26,27}$ Just through ceiling culture and without the addition of cytokines, isolated adipocytes can undergo dedifferentiation and acquire multilineage differentiation potency. ${ }^{28,29}$ However, it is still unclear whether this phenomenon is a hallmark of cell differentiation programs or displays specificities depending on the stem cell types.

Despite being morphologically and phenotypically similar to uncommitted PDMSCs, DePDMSCs represent a previously undescribed distinct population of stem cells with several distinguished features. A previous study showed that the expression levels of ESCs markers were gradually upregulated after dedifferentiation of adipogenic amniotic fluid stem cells. ${ }^{30}$ Generally speaking, stem cells became static and had nearly no proliferative activity after differentiation. It is generally accepted that decreased cell-division ability accompanies long culture times and increased age. ${ }^{31}$ Considering the intriguing results in our study, AL cells may return to the cell 
cycle and proliferate quickly after dedifferentiation, indicating that this proliferative restriction is not permanent and dedifferentiation activates cell cycle progression genes for subsequent proliferation and transdifferentiation. ${ }^{15}$
Recent studies performed on ESCs and iPSCs showed enhanced mitochondrial biogenesis after differentiation, ${ }^{18}$ but whether the mitochondrial biogenesis in various stem cell models is similar to ESCs or iPSCs is not clear. Mitochondria a

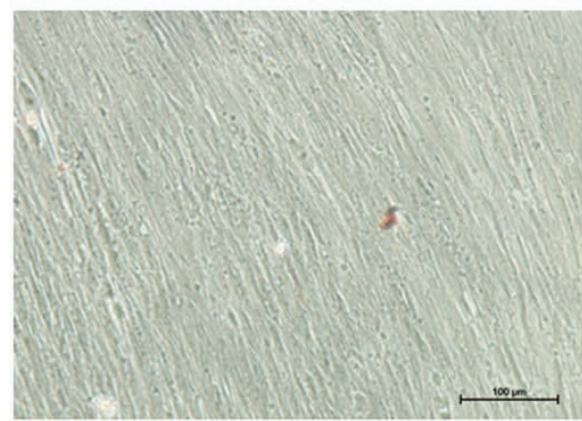

C

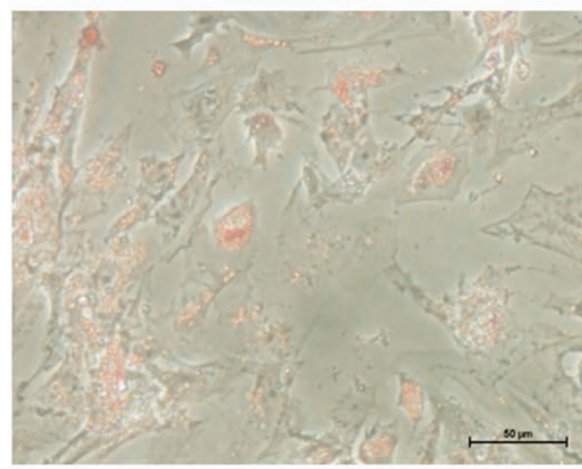

b

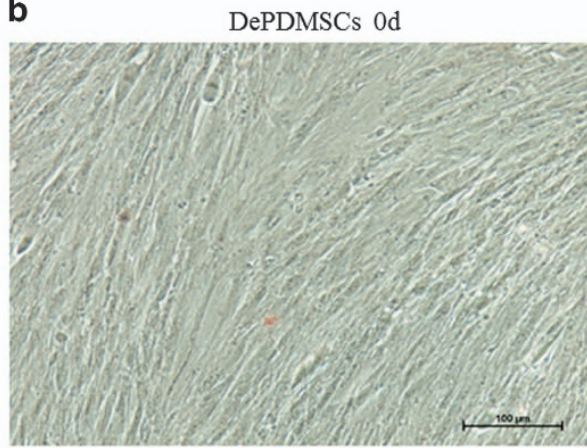

d

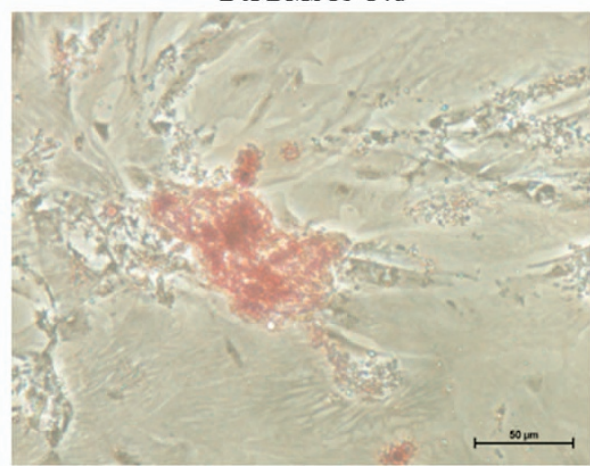

e
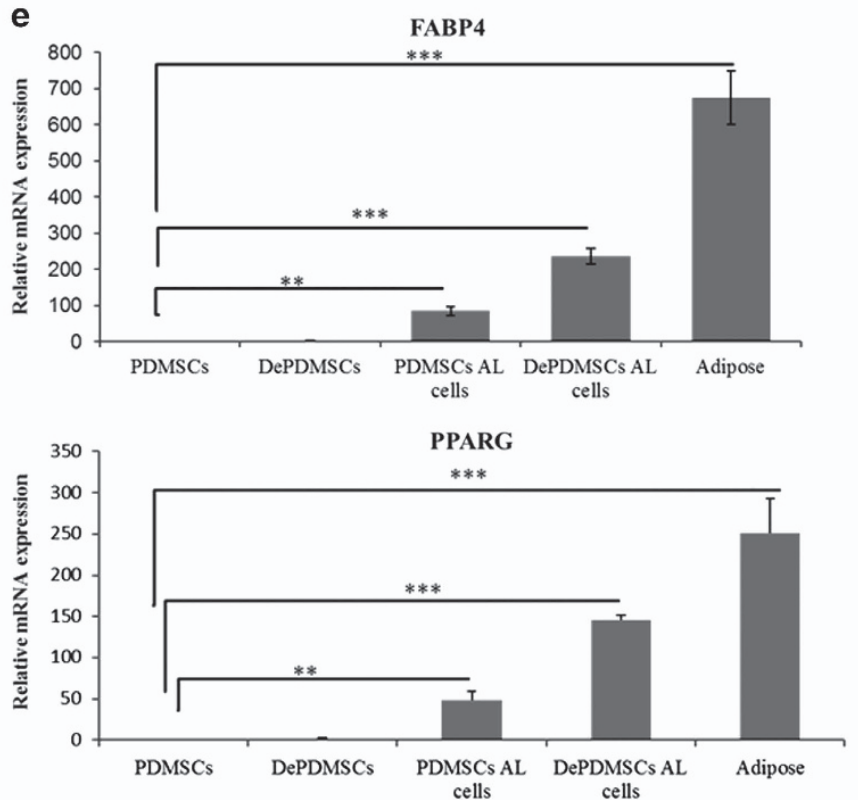

Figure 5 Adipogenic differentiation ability in DePDMSCs showed a greater trend than PDMSCs. (a) Oil red O staining of PDMSCs maintained in normal growth medium; scale bar, $100 \mu \mathrm{m}$. (b) Oil red $\mathrm{O}$ staining of DePDMSCs maintained in normal growth medium; scale bar, $100 \mu \mathrm{m}$. (c) Oil red O staining of PDMSCs that had undergone adipogenic differentiation at 14d (lipid accumulation); scale bar, $50 \mu \mathrm{m}$. (d) Oil red $\mathrm{O}$ staining of DePDMSCs that had undergone adipogenic differentiation at 14d (lipid accumulation); scale bar, $50 \mu \mathrm{m}$. (e) Representative RT-QPCR analyses of PDMSCs and DePDMSCs cultured in adipogenic medium for 14 days. Human adipose tissue was used as a positive control. The expression levels of FABP4 and PPARG in PDMSCs were normalized to 1. (f) Immunocytochemistry of FABP4 and PPARG showed negative expression in PDMSCs and DePDMSCs on day 0 and strongly positive expression on day 14; scale bar, $50 \mu \mathrm{m}$. Data are presented as the mean \pm S.D. in triplicate and were statistically analyzed by one-way ANOVA ( $n=3$ independent donor cells); ${ }^{* \star} P<0.01,{ }^{* \star *} P<0.001$ 
$\mathbf{f}$
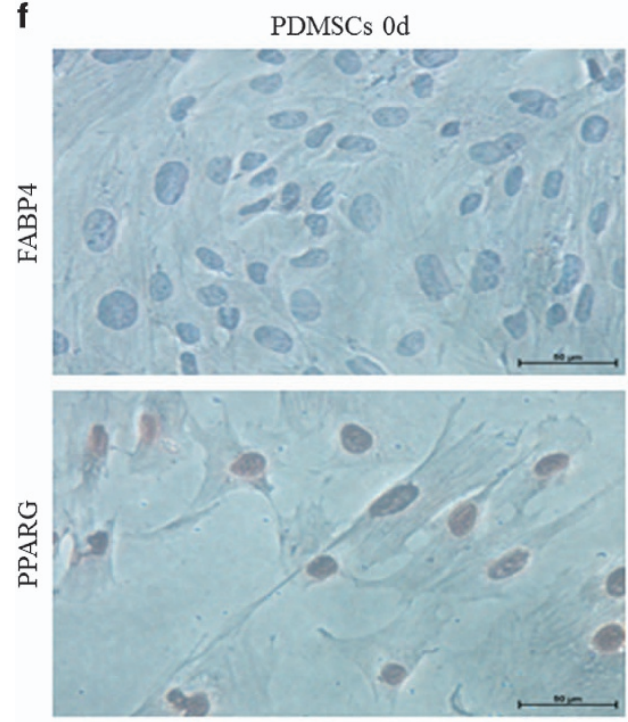

DePDMSCs 0d
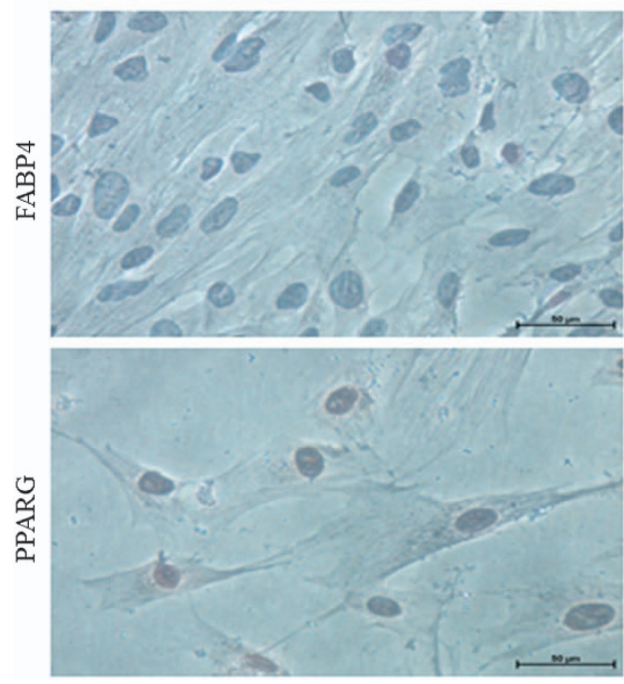
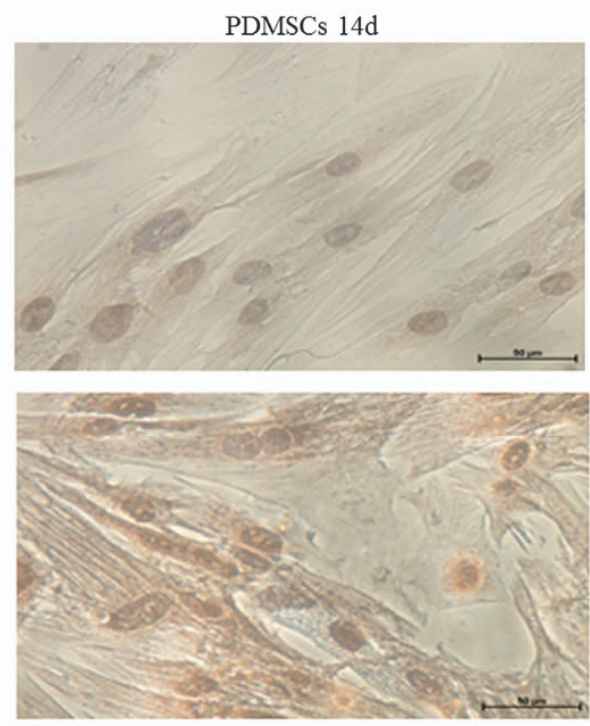

DePDMSCs 14d
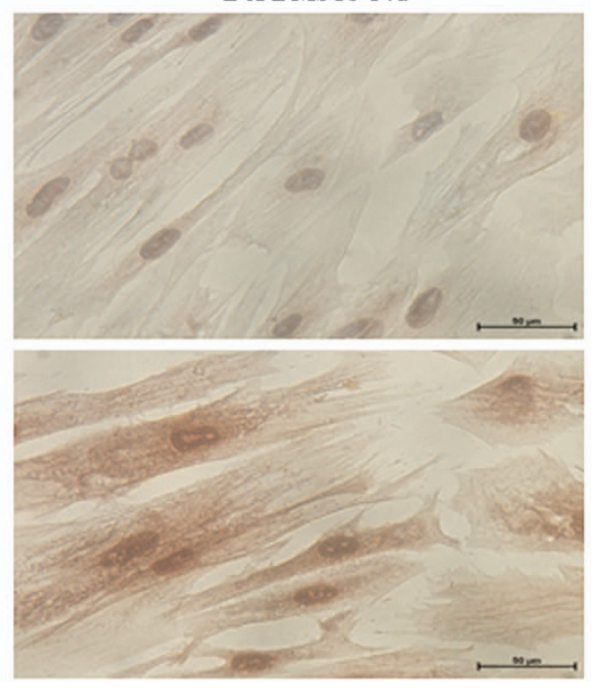

Figure 5 Continued

are cytoplasmic organelles that have a primary role in cellular metabolism and homeostasis, the regulation of the cell signaling and programmed cell death. ${ }^{32}$ Some reports discovered a similar phenomenon in MSCs, but this was limited to BMMSCs. ${ }^{33-38}$ Studies of adipogenic or osteogenic differentiation of BMMSCs also showed an increase in mitochondrial biogenesis and function (an increased mRNA abundance for MT-CO1, MT-CO2, COX4I1 and ATP5A1) during early steps of the differentiation process. ${ }^{36,39}$ Furthermore, evidence also indicates that mitochondrial biogenesis is strongly associated with differentiation, and is accompanied with increased expression of subunits from complexes I, II and III, and a higher mitochondrial activity by significantly increased oxygen consumption. ${ }^{40}$ As a consequence, differentiated cells displayed increased mitochondrial mass, a more developed mitochondrial network, and a shift toward OXPHOS to meet their energy demands. ${ }^{41-43}$ Besides, NNT functions as a high-capacity source of mitochondrial nicotinamide adenine dinucleotide phosphate (NADPH), the mutation results in mitochondrial redox abnormalities, most notably a poor ability to sustain NADP and glutathione in their reduced states, ultimately resulting in increased cellular oxidative stress and impaired morphology and mitochondrial function. ${ }^{44,45}$ These co-regulators cooperate to drive mitochondrial biogenesis and oxidative switching by co-activating many transcription factors. In fact, the use of molecules to promote or inhibit mitochondrial biogenesis or function, or by interfering with the expression of mitochondrial biogenesis regulators or proteins involved in mitochondrial function, has been demonstrated to impact stemness and cell differentiation. ${ }^{18,46,47}$

To determine whether the converted cells with similar phenotypes are just structural entities or retain their multilineage potency requires further investigation. Then, we converted DePDMSCs to adipocytes, osteocytes and hepatocytes. A previous study showed that the dedifferentiated 
a

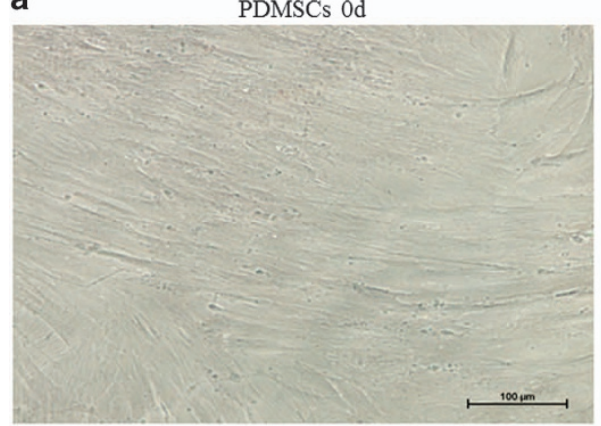

C

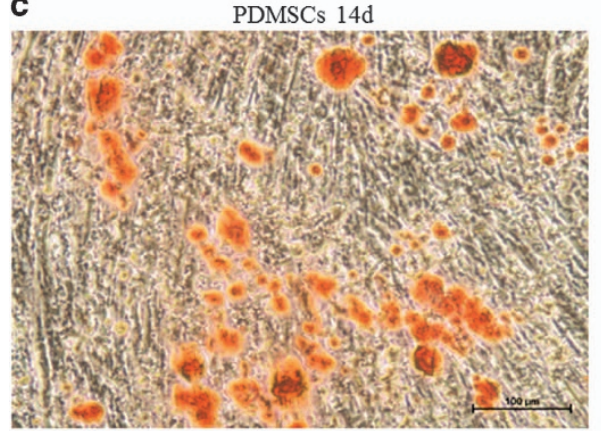

b

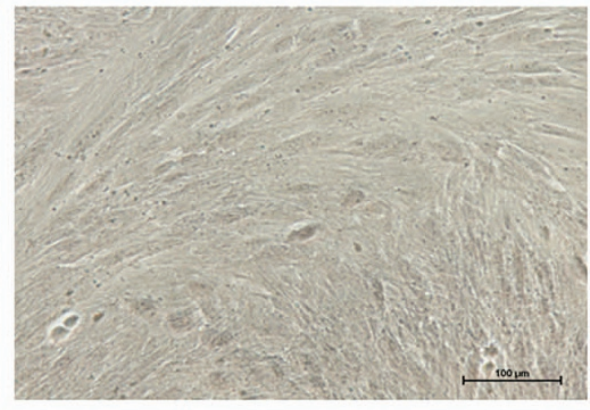

d

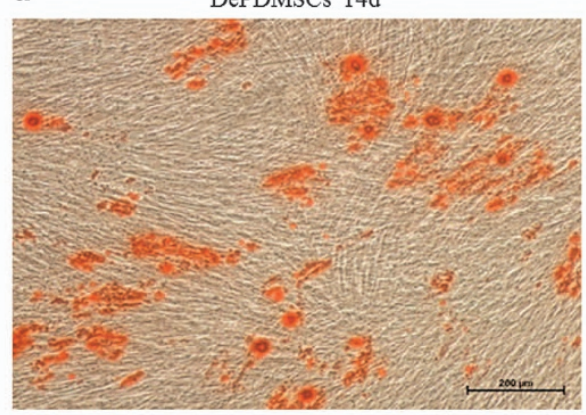

e

RUNX2

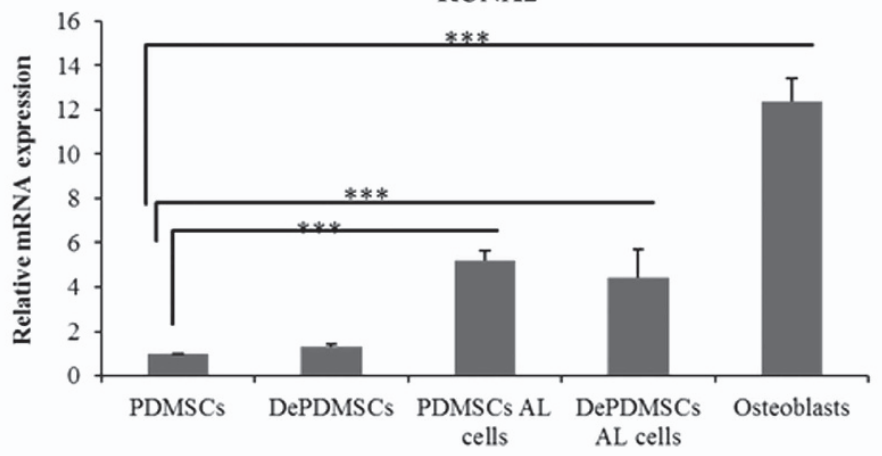

Osteocalcin

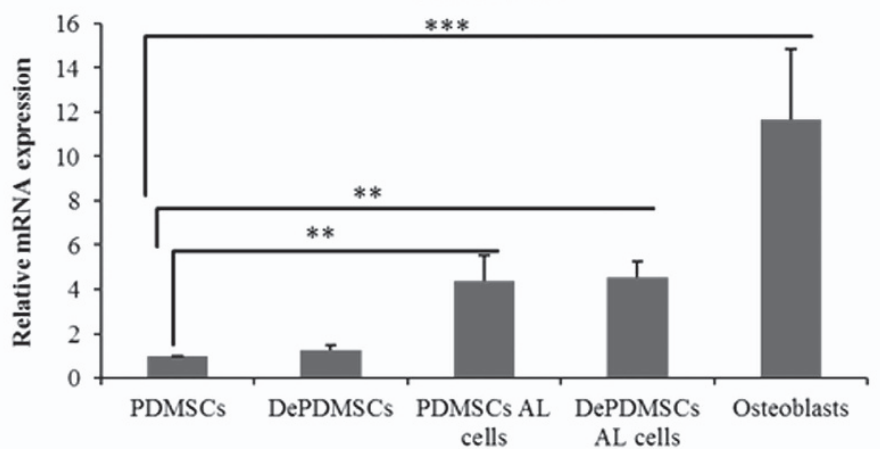

Figure 6 The osteogenic differentiation ability in DePDMSCs was comparable to PDMSCs. (a) Alizarin red staining of PDMSCs maintained in normal growth medium; scale bar, $100 \mu \mathrm{m}$. (b) Alizarin red staining of DePDMSCs maintained in normal growth medium; scale bar, $100 \mu \mathrm{m}$. (c) Alizarin red staining of PDMSCs that had undergone osteogenic differentiation at 14d (bone nodules formed); scale bar, $100 \mu \mathrm{m}$. (d) Alizarin red staining of DePDMSCs that had undergone osteogenic differentiation at 14d (bone nodules formed); scale bar, $200 \mu \mathrm{m}$. (e) Representative RT-QPCR analyses of PDMSCs and DePMSCs cultured in osteogenic medium for 14 days. Osteoblasts were used as a positive control. The expression levels of RUNX2 and OC in PDMSCs were normalized to 1. (f) Immunocytochemistry of RUNX2 and OC showed negative expression in PDMSCs and DePDMSCs on day 0 and strongly positive expression on day 14 ; scale bar, $50 \mu \mathrm{m}$. Data are presented as the mean \pm S.D. in triplicate and were statistically analyzed by one-way ANOVA ( $n=3$ independent donor cells); ${ }^{* *} P<0.01,{ }^{* * *} P<0.001$ 
f
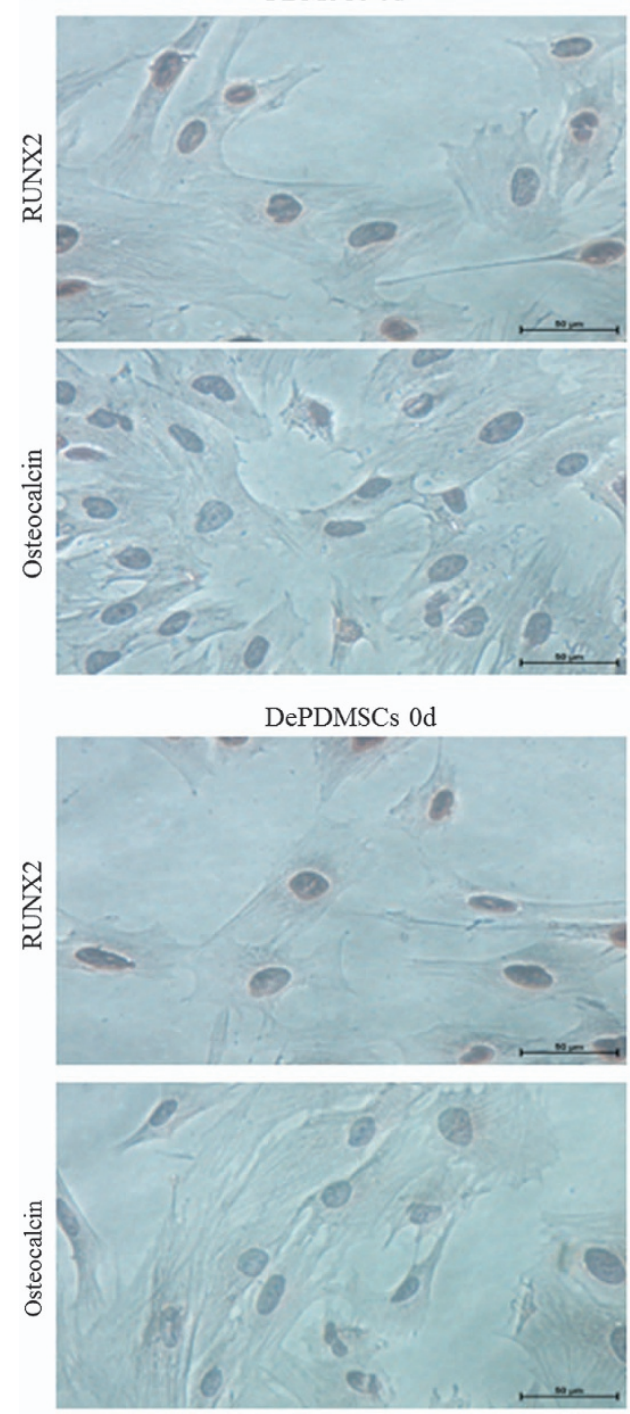

PDMSCs 14d
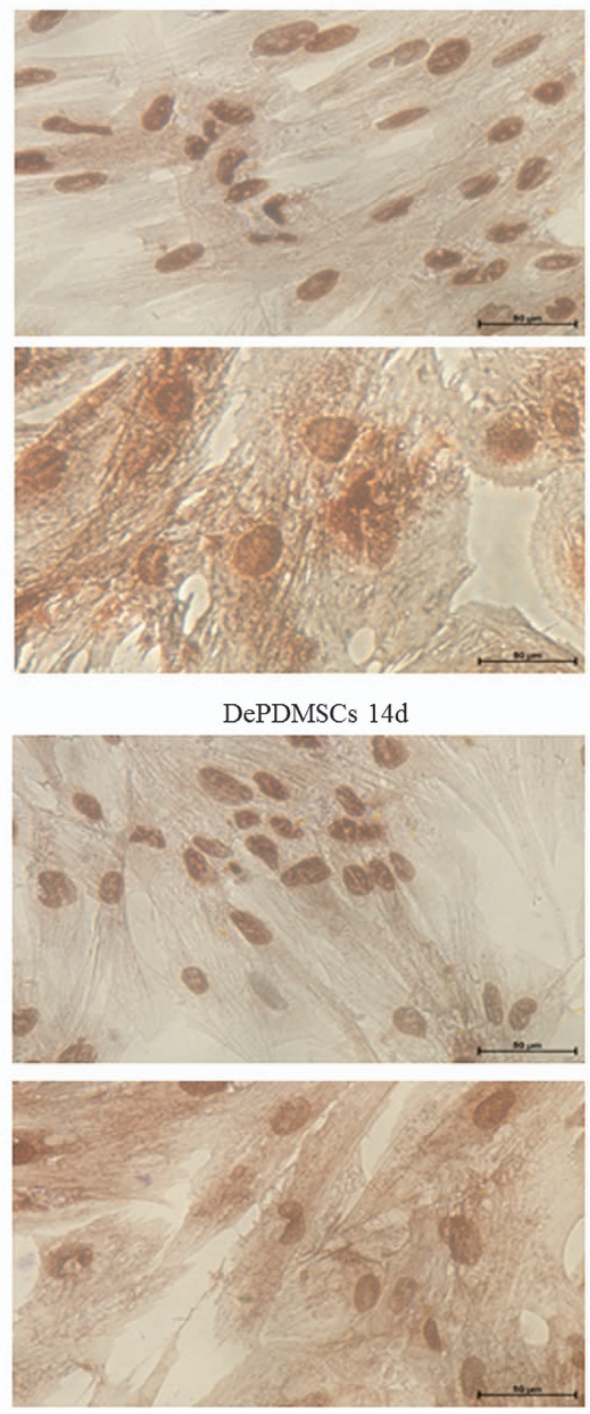

Figure 6 Continued

adipocytes had adipogenic potency, ${ }^{29}$ the results of our study suggest greater adipogenic potency in DePDMSCs than in PDMSCs. Furthermore, another study demonstrated that the dedifferentiated cells could achieve the morphology of other lineages more easily and quickly during the process of transdifferentiation. ${ }^{48}$ On the other hand, dedifferentiated BMMSCs can redifferentiate into neural cells, osteocytes and adipocytes, ${ }^{15,16}$ the dedifferentiated PDMSCs in our study were able to overcome the mesodermal commitment to other lineages. However, there is no study on the detection of hepatocyte differentiation potency in dedifferentiated MSCs as far as we know. The hepatogenic differentiation ability of DePDMSCs is comparable to PDMSCs in our study. In sum, the successful fate conversion of dedifferentiated PDMSCs was not restricted to related lineages within the same germ layer but notably crossed the lineage boundaries beyond limited cellular conversion. ${ }^{49}$
The sequential adipogenic differentiation and dedifferentiation processes resulted in novel stem cells that proliferated faster and retained multilineage potency; however, the mechanism underlying this cross-talk remained to be determined. In this context, we identified a number of differentially expressed genes that were regulated by the complex communication between signaling pathways during adipogenic differentiation and dedifferentiation. Intriguingly, we found a single gene named CADM1 was extremely higher in DePDMSCs when compared with PDMSCs. CADM1 can directly regulate mast cell net adhesion directly through CADM1-dependent adhesion, ${ }^{25}$ furthermore, it was strongly correlated with the bone-forming capacity of human MSCs and could be used as a reliable in vitro diagnostic marker. ${ }^{50} \mathrm{CAMs}$ serve as a well-known signaling pathway for diverse biological processes including cellular interactions, adhesions and micro-environmental decisions. ${ }^{22}$ In addition to that, ECM 
has a critical role in the formation of adipogenically differentiated cells and the differentiated cells started to release the lipid droplets and leave bare network of
ECM. ${ }^{51,52}$ The ECM components modulate cell behavior and enable the cells to regenerate, proliferate, differentiate, grow, orientate and constrain themselves for perfectible a

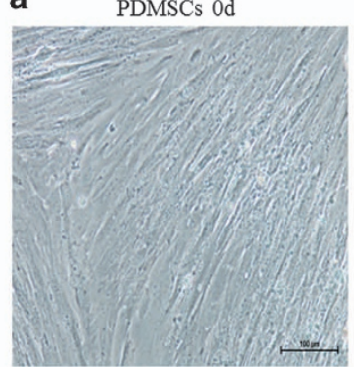

d

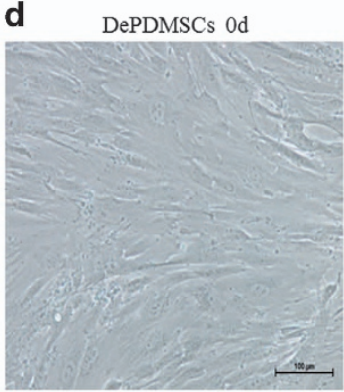

b

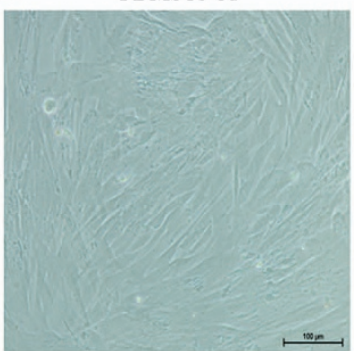

e DePDMSCs $8 \mathrm{~d}$
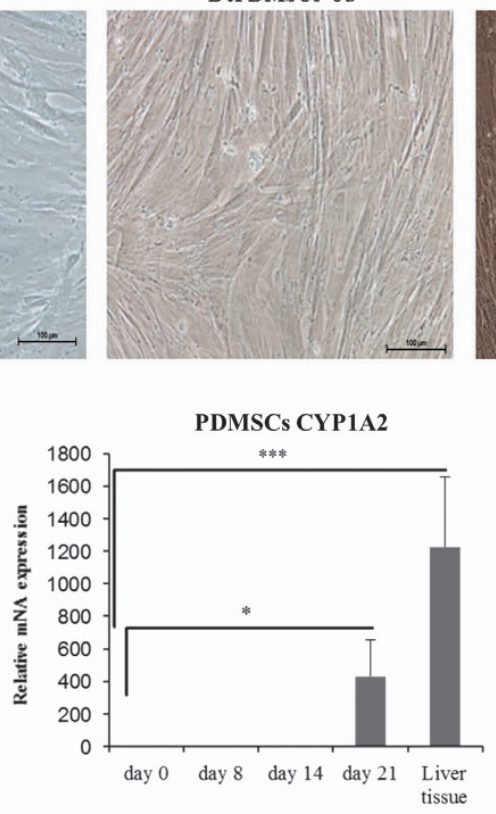

PDMSCs CYP1A2

DePDMSCs CYP1A2

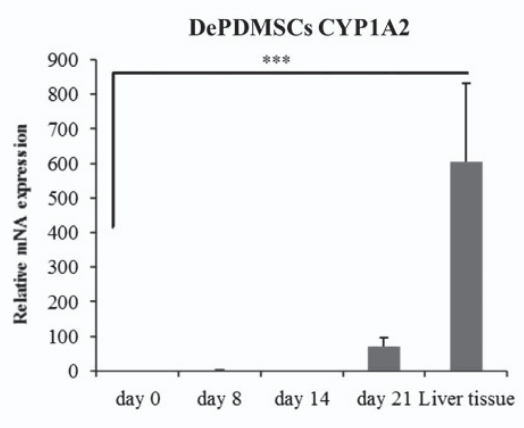

C $\quad$ PDMSCs $21 \mathrm{~d}$
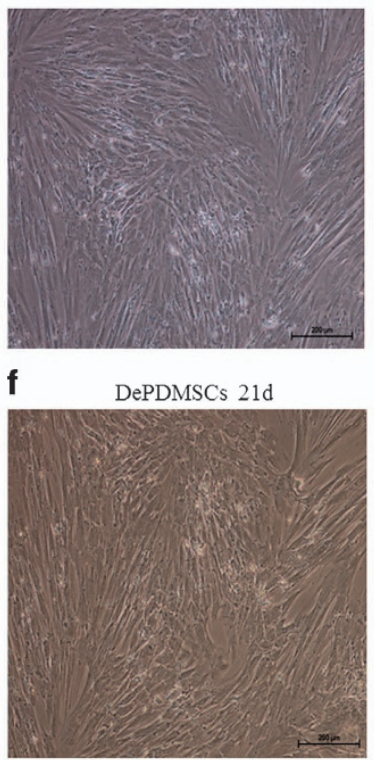

g

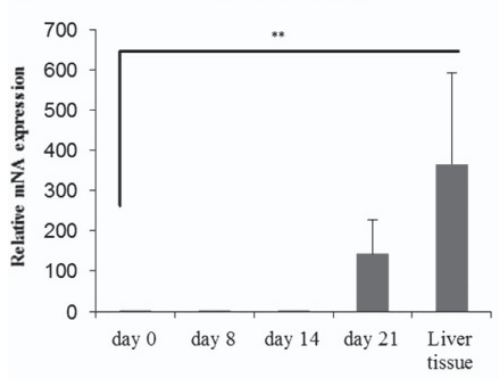

DePDMSCs ALB

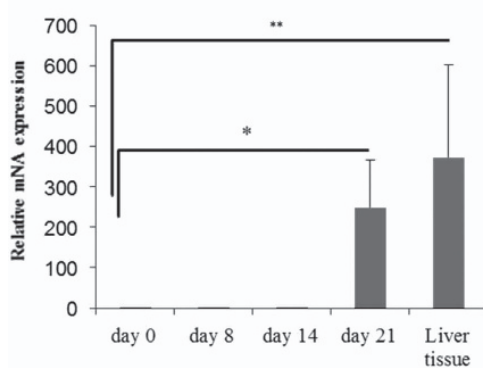

PDMSCs CYP3A4

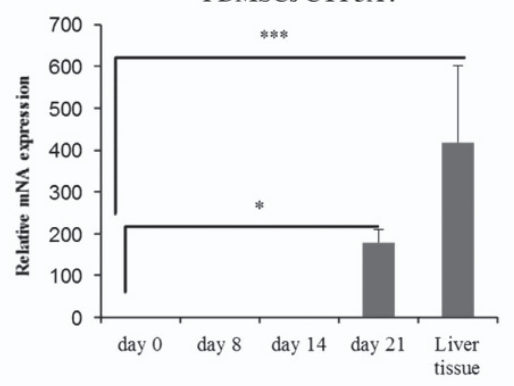

DePDMSCs CYP3A4

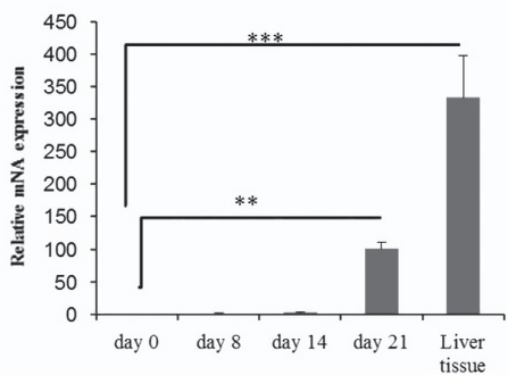

Figure 7 The hepatogenic differentiation ability in DePDMSCs was comparable to PDMSCs. (a) Hepatocyte differentiation of PDMSCs at day 0; scale bar, $100 \mu \mathrm{m}$. (b) Hepatocyte differentiation of PDMSCs at day 8; scale bar, $100 \mu \mathrm{m}$. (c) Hepatocyte differentiation of PDMSCs at day 21; scale bar, $200 \mu \mathrm{m}$. (d) Hepatocyte differentiation of DePDMSCs at day 0; scale bar, $100 \mu \mathrm{m}$. (e) Hepatocyte differentiation of DePDMSCs at day 8; scale bar, $100 \mu \mathrm{m}$. (f) Hepatocyte differentiation of DePDMSCs at day 21; scale bar, $200 \mu \mathrm{m}$. (g) RT-QPCR analysis of ALB, CYP1A2 and CYP3A4 in hepatogenic PDMSCs and DePDMSCs on days 0, 8, 14 and 21. Liver tissue was as a positive control. The undifferentiated PDMSC group or undifferentiated DePDMSCs was normalized to 1. The mRNA expression levels of CYP1A2 and CYP3A4 in PDMSCs were significantly increased after hepatogenic induction for $21 \mathrm{~d}(P<0.05)$. The mRNA expression levels of ALB $(P<0.05)$ and CYP3A4 $(P<0.01)$ in DePDMSCs were significantly increased after hepatogenic induction for 21 d. Data are presented as the mean \pm S.D. in triplicate and were statistically analyzed by one-way ANOVA ( $n=3$ independent donor cells); $P<0.05$, ${ }^{* *} P<0.01,{ }^{* \star} P<0.001$. (h) Immunocytochemistry of AFP, CK18 and CK19 showed negative expression in PDMSCs at day 0 , weakly positive expression at day 8 , and strongly positive expression at day 21. (i) Immunocytochemistry of AFP, CK18 and CK19 showed negative expression in DePDMSCs at day 0, weakly positive expression at day 8 and strongly positive expression at day 21; scale bars, $100 \mu \mathrm{m}$. (j) Uptake of ICG on hepatogenic PDMSCs and DePDMSCs at day 0 and day 21 and secretion of ICG from hepatogenic PDMSCs and DePDMSCs at day 21; scale bars, $100 \mu \mathrm{m}$. (k) Periodic acid staining for glycogen on hepatogenic PDMSCs and DePDMSCs at day 0 (scale bar, $200 \mu \mathrm{m}$ ) and day 21 (scale bar, $50 \mu \mathrm{m})$ 

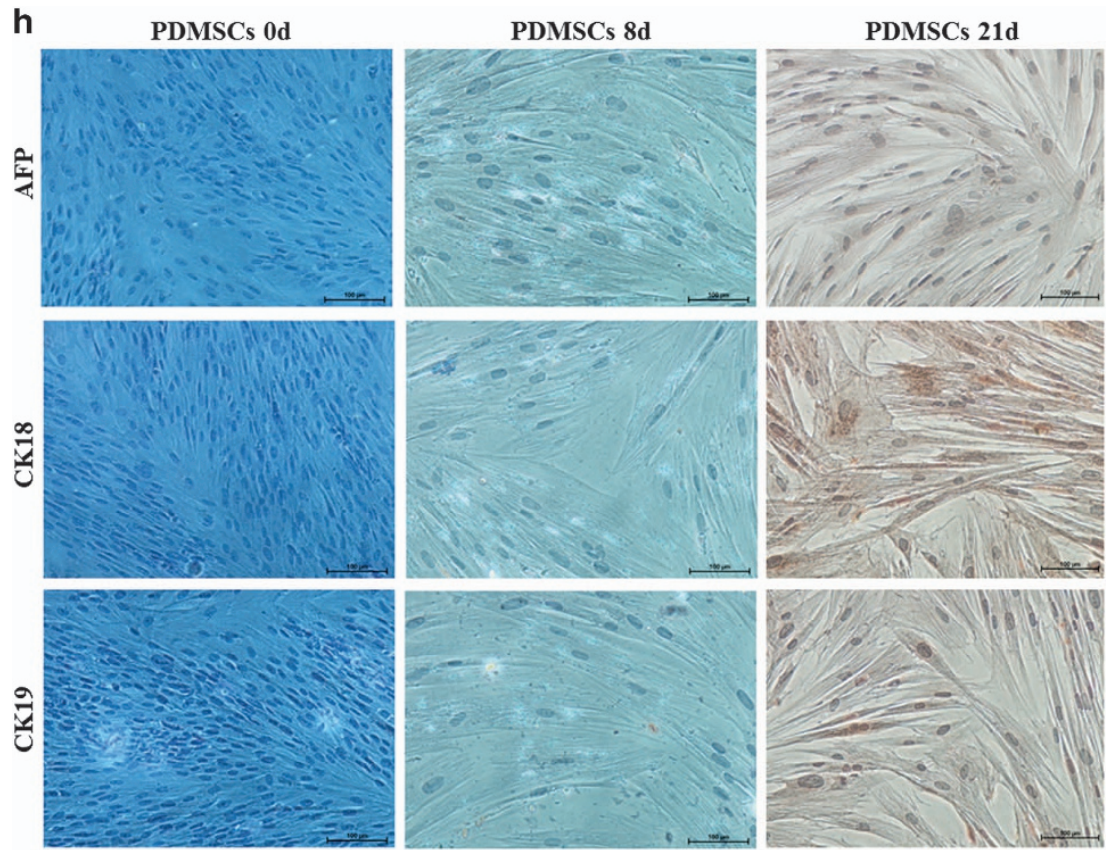

i
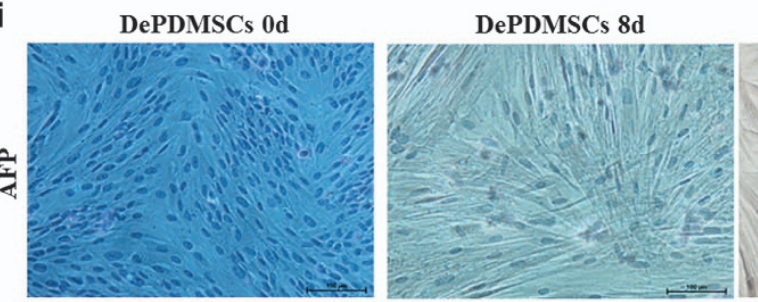

DePDMSCs 21d
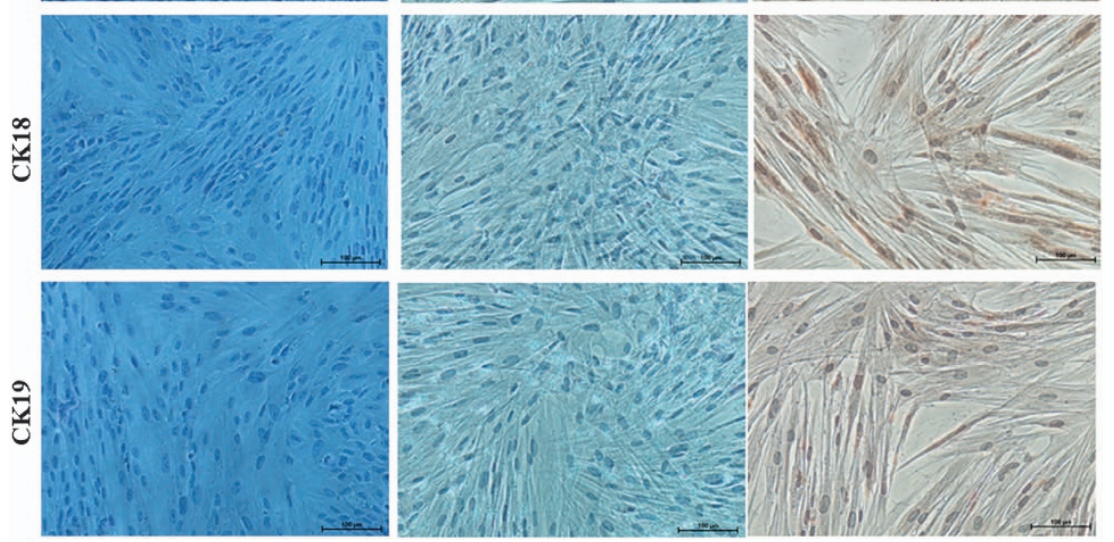

Figure 7 Continued

regeneration by cell-cell and cell-ECM interactions. ${ }^{23}$ Here, we indicated that the enhanced proliferative ability and differentiation ability of DePDMSCs may attribute to the upregulated CAMs signaling pathway and ECM components. In spite of that, further research is still required to unravel the process in other cell types and to clarify more detailed mechanisms involved in the interplay between these two processes, which progressed as a reprogramming method in ESCs or iPSCs.

In conclusion, we systematically and comprehensively demonstrated that PDMSC-derived AL cells were able to successfully dedifferentiate and acquire a more primitive phenotype under certain culture conditions. In addition, we not only detected alterations in morphology, adipocyte markers and stem cell markers, but we also observed the mitochondrial network during differentiation and dedifferentiation and found that it may serve as a marker of absent or acquired pluripotency in various stem cell models. Furthermore, the dedifferentiated cells entered the cell cycle and retained their multi-potentiality to transdifferentiate into other lineages in response to extrinsic factors. The CAMs signaling pathway and ECM components regulate cell behavior in proliferation and differentiation; consequently, we suggest that biochemical analysis of native adipogenic ECM would be a 

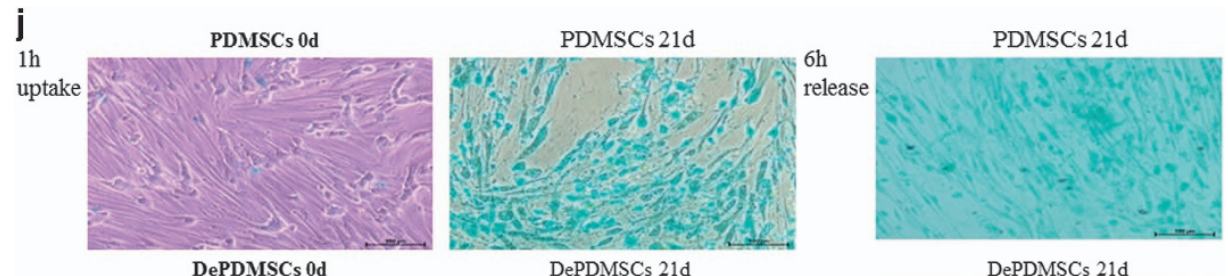

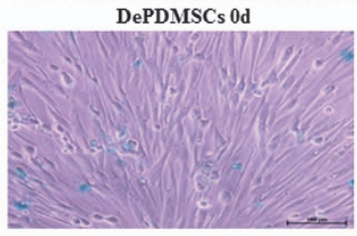

DePDMSCs 21d
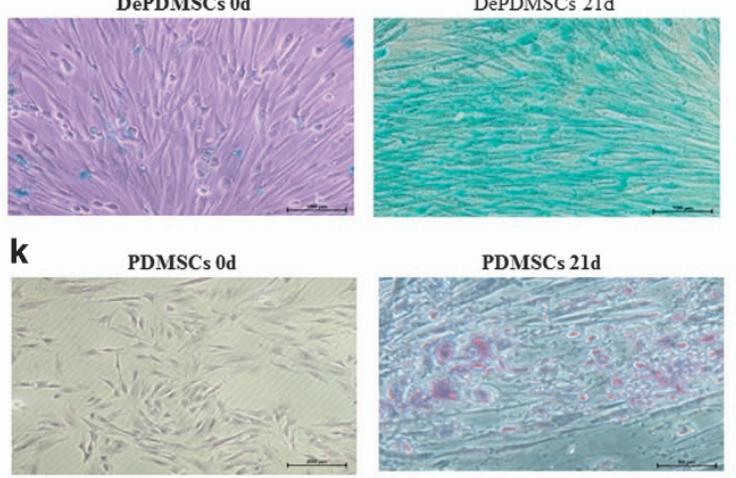

DePDMSCs 0d

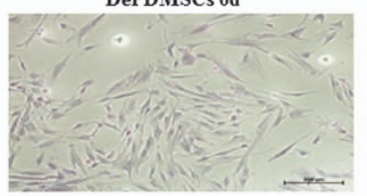

PDMSCs 21d

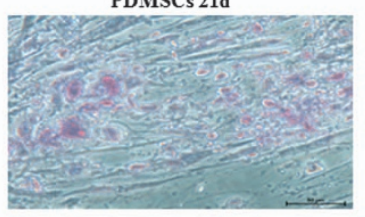

DePDMSCs $21 d$

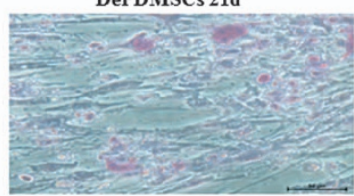

Figure 7 Continued

crucial guide for artificial designing of biomaterials. With respect to the clinical use of MSCs in regenerative medicine, the sequence differentiation, dedifferentiation, redifferentiation and transdifferentiation could be a vital approach to treat damaged or diseased tissues or to generate tissue-specific mature cells in vitro in the future.

\section{Materials and Methods}

Isolation and culturing of PDMSCs. Placentas were obtained from donors at the Hangzhou Red Cross Hospital in China after informed consent had been obtained. The study used a protocol approved by the Research Ethics Committee of the First Affiliated Hospital, School of Medicine, Zhejiang University. The placental tissue was washed with preheated phosphate-buffered saline (PBS, pH 7.2 \pm 0.1 , GenomSciences, Hangzhou, China), minced and digested using $0.1 \%(\mathrm{w} / \mathrm{v})$ collagenase type IV (Invitrogen Life Technologies, Carlsbad, CA, USA) at $37^{\circ} \mathrm{C}$ for $30 \mathrm{~min}$. Recovered cells and digested cell debris were filtered through a $100-\mu \mathrm{m}$ cell strainer. Mononuclear cells obtained by lymphocyte isolation (GE Healthcare, Ficoll-Pague PLUS, Uppsala, Sweden) were cultured in special medium (MesenCult Human Basal Medium plus MesenCult Human Supplement, STEMCELL Technologies, Vancouver, BC, Canada) and adjusted to $2 \times 10^{6}$ cells in T25-cm2 tissue culture flasks (Nunc Flasks Nunclon $\Delta$ with Filter Cap, Nunclon $\triangle$ Surface, Roskilde, Denmark) maintained in an incubator at $37^{\circ} \mathrm{C}$ in a humidified atmosphere with $5 \%(\mathrm{v} / \mathrm{v}) \mathrm{CO}_{2}$. Approximately 4 to 6 days later, many colonies had formed. After achieving 60-70\% confluence, adherent cells were trypsinized with $0.25 \%(\mathrm{w} / \mathrm{v})$ trypsin/EDTA (Invitrogen Life Technologies) and re-plated at a 1:3 dilution. Placenta-derived cells were cultured continuously under the same conditions.

Adipogenic differentiation and dedifferentiation of PDMSCs. To induce adipogenesis, the third passage PDMSCs at $1 \times 10^{5} /$ well density in 12-well plates were treated with adipogenic medium (OriCell hMSC Adipogenic Differentiation Medium, Cyagen Biosciences, Guangzhou, China) in 12-well plates for 2 weeks. The differentiated PDMSCs were defined as AL cells and then the adipogenic medium was removed from AL cells and replaced by general PDMSCs medium for 1 week. After that, the cells were defined as DePDMSCs at passage 1 . Then, DePDMSCs continued to be cultured in general PDMSCs medium to passage 3. After 2 weeks, lipoprotein lipase (FABP4) and peroxisome proliferatoractivated receptor-g (PPARG) were identified by mRNA detection.

RNA extraction and reverse transcription. Using the RNAiso plus kit (TaKaRa, Tokyo, Japan), total RNA was isolated according to the manufacturer's instructions. The RNA was first treated with DNase (TaKaRa) in a $10 \mu$ reaction with $5 \times \mathrm{gDNA}$ Easer Buffer $(2 \mu \mathrm{l})$, gDNA Easer $(1 \mu \mathrm{l})$ and total RNA $(1 \mu \mathrm{g})$. The reaction was conducted at $42^{\circ} \mathrm{C}$ for $2 \mathrm{~min}$. For the mRNAs, the PrimeScript RT reagent Kit (TaKaRa) was used for reverse transcription (RT) in a total volume of $20 \mu$ l with $4 \mu \mathrm{l}$ $5 \times$ PrimeScript Buffer PCR buffer, $1 \mu$ l PrimeScript RT enzyme mix I, $1 \mu \mathrm{L}$ RT Primer Mix and $10 \mu \mathrm{l}$ of the RNA sample. The RT reaction started with a 15-min incubation period at $37^{\circ} \mathrm{C}$ and ended after a 5 -s enzyme-denaturing step at $85^{\circ} \mathrm{C}$.

Real-time quantitative-PCR. Real-time amplification was performed using the SYBR Premix Ex Taq (TaKaRa) in an ABI 7900 thermocycler (Applied Biosystems, Foster City, CA, USA). Real-time quantitative-PCR (RT-QPCR) was carried out in a $10 \mu$ l reaction system containing $5 \mu$ l SYBR Premix Ex Taq $(2 \times)$, $0.4 \mu \mathrm{l}$ PCR primers $(10 \mu \mathrm{M}), 0.2 \mu \mathrm{l}$ ROX reference dye and $1 \mu \mathrm{l}$ cDNA. PCR thermal cycling parameters were $95^{\circ} \mathrm{C}$ for $30 \mathrm{~s}$, followed by 40 cycles of $95^{\circ} \mathrm{C}$ for $5 \mathrm{~s}$ and then $60^{\circ} \mathrm{C}$ for $30 \mathrm{~s}$. After PCR, a dissociation curve was constructed at $95^{\circ} \mathrm{C}$ for $15 \mathrm{~s}, 60^{\circ} \mathrm{C}$ for $15 \mathrm{~s}$ and $95^{\circ} \mathrm{C}$ for $15 \mathrm{~s}$ for detection of PCR product specificity. Each reaction was performed in triplicate. Positive mesenchymal surface antigens and mitochondria, adipocyte, osteocyte and hepatocyte-specific genes were performed by RT-QPCR. The mitochondrial specific genes detected included ATP synthase, $\mathrm{H}_{+}$transporters, mitochondrial $\mathrm{F} 1$ complex, alpha subunit 1 (ATP5A1), cytochrome $c$ oxidase subunit IV isoform 1 (COX4I1), mitochondrially encoded cytochrome $c$ oxidase I (MT-CO1), mitochondrially encoded cytochrome $c$ oxidase II (MT-CO2), transcription factor A, mitochondrial (TFAM), translocase of outer mitochondrial membrane 34 (TOMM34), lon peptidase 1, mitochondrial (LONP1) and peroxisome proliferator-activated receptor alpha (PPAR-a). The adipocyte-specific genes including FABP4 and PPARG were detected. The osteocyte-specific genes including runt-related transcription factor 2 (RUNX2) and osteocalcin (OC) were detected. The hepatocyte-specific genes including 
cytochrome $\mathrm{P}$ (CYP)1A2, CYP3A4 and albumin (ALB) were detected. The differentially expressed genes of microarray in DePDMSCs and PDMSCs were confirmed by RT-QPCR. The reference gene $\beta$-actin was used as a relative control for the expression levels. The primers for the target products were designed as in Supplementary Table S4.

Table 1a The enriched KEGG pathway of down- and upregulated differentially expressed genes in PDMSCs and AL cells (top five in each)

\begin{tabular}{|c|c|c|c|c|}
\hline Category & Description & Count & $\%$ & $P$-value \\
\hline \multicolumn{5}{|c|}{ Downregulated } \\
\hline KEGG & Focal adhesion & 42 & 3.7466548 & $1.18 \mathrm{E}-09$ \\
\hline KEGG & $\begin{array}{l}\text { Regulation of actin } \\
\text { cytoskeleton }\end{array}$ & 41 & 3.6574487 & $3.06 \mathrm{E}-08$ \\
\hline KEGG & Pathways in cancer & 33 & 2.9438002 & 0.0648822 \\
\hline KEGG & $\begin{array}{l}\text { MAPK signaling } \\
\text { pathway }\end{array}$ & 31 & 2.765388 & 0.0137503 \\
\hline KEGG & $\begin{array}{l}\text { Chemokine } \\
\text { signaling pathway }\end{array}$ & 22 & 1.9625335 & 0.0360058 \\
\hline \multicolumn{5}{|c|}{ Upregulated } \\
\hline KEGG & Pathways in cancer & 26 & 2.8571429 & 0.0240712 \\
\hline KEGG & Lysosome & 16 & 1.7582418 & $7.22 \mathrm{E}-04$ \\
\hline KEGG & $\begin{array}{l}\text { Insulin signaling } \\
\text { pathway }\end{array}$ & 13 & 1.4285714 & 0.0392537 \\
\hline KEGG & $\begin{array}{l}\text { Jak-STAT signaling } \\
\text { pathway }\end{array}$ & 13 & 1.4285714 & 0.0914349 \\
\hline KEGG & $\begin{array}{l}\text { Adipocytokine } \\
\text { signaling pathway }\end{array}$ & 12 & 1.3186813 & $4.57 E-04$ \\
\hline
\end{tabular}

Flow cytometry for surface antigen expression. Culture-expanded cells were washed with PBS-containing $0.3 \%(\mathrm{w} / \mathrm{v})$ bovine serum albumin (BSA) and the concentration was adjusted to $1 \times 10^{6}$ cells $/ 100 \mu$ l. PDMSCs at passage 3 , AL cells and DePDMSCs at passage 3 were examined for mesenchymal and hematopoietic marker expression of surface antigens by incubating with the antibodies CD14-phycoerythrin (PE), CD29-fluorescein isothiocyanate (FITC), CD44-FITC, CD105-PE and HLA-DR-FITC (Abcam, Cambridge, UK). Antibodies including mouse IgG2a-FITC, mouse IgG2a-PE/Cy5.5, mouse IgG1-FITC and rat IgG2b-FITC (Abcam) were used as isotype controls. After being labeled with antibodies in the dark at room temperature for $30 \mathrm{~min}$, cells were washed twice with PBS. Flow cytometry was conducted using a BD LSR II (Beckman Coulter, Los Angeles, CA, USA), and the data were analyzed using BD FACSDiva software.

Mitochondria fluorescent measurement. Mito-Tracker Green FM (Invitrogen Life Technologies) was diluted in L-DMEM (Gibco, Carlsbad, CA, USA) to the appropriate concentrations. Cells were incubated with pre-warmed $\left(37^{\circ} \mathrm{C}\right.$ ) probe containing medium for $60 \mathrm{~min}$ for staining. PDMSCs at passage 3 , AL cells and DePDMSCs at passage 3 were visualized and photographed on a fluorescent microscope (ZEISS LSM710, Jena BioSciences, Jena, Germany). Five different areas from each image were taken.

Cell lysis collection. Cells were rinsed with ice-cold PBS, and then ice-cold mitochondrial lysis buffer with freshly added phosphatase and protease inhibitors were added ( $0.2 \mathrm{ml}$ per well of a 12 -well plate). Adherent cells were scraped off the dish with a cell scraper and the suspension was transferred into a centrifuge tube and gently rocked for $15-30 \mathrm{~min}$ at $4^{\circ} \mathrm{C}$. The lysate was centrifuged at $14000 \times \mathrm{g}$ for $20 \mathrm{~min}$ at $4{ }^{\circ} \mathrm{C}$ and the supernatant was immediately transferred into fresh prechilled micro-centrifuge tubes. The lysate was diluted at 1:4 for BCA assays with a spectrophotometer (Beckman Coulter Multimode Detector DTX880, Beckman Coulter). Finally, the lysate was aliquoted and stored at $\leqslant-70^{\circ} \mathrm{C}$.

Table 1b The enriched KEGG pathway of down- and upregulated differentially expressed genes in AL cells and DePDMSCs (top five in each)

\begin{tabular}{|c|c|c|c|c|}
\hline Category & Description & Count & $\%$ & $P$-value \\
\hline \multicolumn{5}{|l|}{ Downregulated } \\
\hline KEGG & Pathways in cancer & 26 & 3.044496487 & 0.043737346 \\
\hline KEGG & Lysosome & 17 & 1.990632319 & $4.34 \mathrm{E}-04$ \\
\hline KEGG & Adipocytokine signaling pathway & 12 & 1.405152225 & $7.38 \mathrm{E}-04$ \\
\hline KEGG & TGF-beta signaling pathway & 12 & 1.405152225 & 0.006228585 \\
\hline KEGG & Arginine and proline metabolism & 9 & 1.053864169 & 0.006559557 \\
\hline \multicolumn{5}{|l|}{ Upregulated } \\
\hline KEGG & Focal adhesion & 49 & 3.26449034 & $3.56 \mathrm{E}-10$ \\
\hline KEGG & Regulation of actin cytoskeleton & 49 & 3.26449034 & $4.02 E-09$ \\
\hline KEGG & Pathways in cancer & 42 & 2.798134577 & 0.028922984 \\
\hline KEGG & Endocytosis & 27 & 1.798800799 & 0.019686088 \\
\hline KEGG & Tight junction & 23 & 1.532311792 & 0.005755194 \\
\hline
\end{tabular}

Table 1c Three of the nine differentially expressed genes involved in multiple pathways

\begin{tabular}{|c|c|c|c|}
\hline $\begin{array}{l}\text { Gene } \\
\text { symbol }\end{array}$ & Gene name & Species & KEGG_PATHWAY \\
\hline CADM1 & Cell adhesion molecule 1 & Homo sapiens & hsa04514:Cell adhesion molecules (CAMs) \\
\hline FOXO1 & Forkhead box $\mathrm{O} 1$ & Homo sapiens & $\begin{array}{l}\text { hsa04910:Insulin signaling pathway } \\
\text { hsa05200:Pathways in cancer } \\
\text { hsa05215:Prostate cancer }\end{array}$ \\
\hline FGF7 & Fibroblast growth factor 7 & Homo sapiens & $\begin{array}{l}\text { hsa04010:MAPK signaling pathway } \\
\text { hsa04810:Regulation of actin cytoskeleton } \\
\text { hsa05200:Pathways in cancer } \\
\text { hsa05218:Melanoma }\end{array}$ \\
\hline WFDC1 & WAP four-disulfide core domain 1 & Homo sapiens & \\
\hline MMP10 & Matrix metallopeptidase 10 & Homo sapiens & \\
\hline PCDHB2 & Protocadherin beta 2 & Homo sapiens & \\
\hline MAF & v-maf avian musculoaponeurotic fibrosarcoma oncogene homolog & Homo sapiens & \\
\hline ZNF711 & Zinc finger protein 711 & Homo sapiens & \\
\hline SULF2 & Sulfatase 2 & Homo sapiens & \\
\hline
\end{tabular}


Mitochondrial oxidative phosphorylation. Mitochondrial oxidative phosphorylation profiles were determined using the human OXPHOS Magnetic Bead Panel protocol from the Milliplex Map Kit (EMD Millipore, Billerica, MA, USA). Before the assay, the samples were extracted using mitochondrial lysis buffer with protease inhibitors (EMD Millipore) and phosphatase inhibitors (EMD Millipore) according to the recommend protocol. Briefly, OXPHOS assay plates were washed with wash buffer, sealed and mixed on an orbital plate shaker for $10 \mathrm{~min}$ at room temperature. The wash buffer was decanted and $25 \mu \mathrm{l}$ of control, mitochondria lysis buffer and samples were added in each well. Then, $25 \mu \mathrm{l}$ beads were added into each well and incubated for $2 \mathrm{~h}$ at room temperature on an orbital shaker. After incubation, well contents were removed via the washing instructions provided by the protocol. Fifty microliters of detection antibodies were then added to the wells and incubated with samples for $1 \mathrm{~h}$ at room temperature while shaking. After incubation, well contents were removed as previously described and $50 \mu \mathrm{l}$ streptavidinphycoerythrin was added to each well. The samples were incubated with streptavidin-phycoerythrin for $30 \mathrm{~min}$ at room temperature. After the incubation period, samples were washed as previously described and resuspended in Sheath Fluid. Plates were run on the Luminex MagPix machine and data were collected using the Luminex XPONENT software (v. 4.2).

Analysis of cell proliferation. The growth curves of PDMSCs at passage 3 , PDMSCs at passage 6 and DePDMSCs at passage 3 were determined using a Cell Proliferation Reagent WST-1 assay. Each group of cells was seeded at a density of 2000 cells/well in 96-well plates (Nunclon $\Delta$ Surface, Denmark). For 24, 48, 72, 96, 120,144 or $166 \mathrm{~h}, 10 \mu \mathrm{l}$ of WST-1 reagent $(5 \mathrm{mg} / \mathrm{ml}$, Roche, Basel, Switzerland; Russia) was added to each well and cells were incubated for $1 \mathrm{~h}$ at $37^{\circ} \mathrm{C}$ and $5 \%$ (v/v) $\mathrm{CO}_{2}$. Absorbance at $450 \mathrm{~nm}$ was measured using a spectrophotometer (Beckman Coulter Multimode Detector DTX880, Beckman Coulter). Experiments were performed in sextuplicate and repeated at least three times. In cell growth curves, the optical density (OD) values were plotted as a function of the elapsed time.

Differentiation of PDMSCs and DePDMSCs to adipocytes, osteocytes and hepatocytes. To induce adipogenesis, PDMSCs and DePDMSCs of passage 3 at $1 \times 10^{5} /$ well in 12-well plates were treated with adipogenic medium (OriCell hMSC Adipogenic Differentiation Medium, Cyagen Biosciences) as per the manufacturer's protocol. The medium was changed three times per week. After 2 weeks, adipogenesis was confirmed by Oil red $\mathrm{O}$ staining (Sigma, St. Louis, MO, USA). Staining was assessed by bright-field microscopy. FABP4 and PPARG were identified in AL cells, PDMSCs and DePDMSCs at passage 1, DePDMSCs at passage 2 and DePDMSCs at passage 3 by mRNA detection. PDMSCs cultured in normal growth medium and human intraoperative abandoned adipose tissue served as controls.

For osteogenic differentiation, PDMSCs and DePDMSCs at passage 3 at $5 \times 10^{4} /$ well in 12-well plates were treated with osteogenic medium (OriCell hMSC Osteogenic Differentiation Medium, Cyagen Biosciences) as per the manufacturer's protocol. The medium was changed three times per week. After 2 weeks, osteogenic differentiation was evaluated by Alizarin Red S staining (Sigma) and detection of RUNX2 and OC mRNA. PDMSCs cultured in normal growth medium and immortalized human fetal osteoblastic cells (hFOB1.19 cell line, Cell Bank of the Chinese Academy of Sciences, Shanghai, China) served as controls.

For hepatogenic differentiation, PDMSCs and DePDMSCs of passage 3 at $1 \times 10^{5} /$ well in 12-well plates were treated with hepatogenic medium (OriCell hMSC Hepatogenic Differentiation Medium, Cyagen Biosciences) as per the manufacturer's protocol. After induction for 8 and 20 days, hepatogenic differentiation was evaluated by alpha-fetoprotein (AFP), cytokeratin 18(CK18), and cytokeratin 19 (CK19) immunocytochemical staining and detection of ALB, CYP1A2 and CYP3A4 mRNA. PDMSCs cultured in normal growth medium and human liver tissue served as controls.

Immunocytochemistry. Cell samples were fixed in 4\% (v/v) paraformaldehyde solution (MultiSciences Biotech, Hangzhou, China) at room temperature for $15 \mathrm{~min}$. Endogenous peroxidase was inhibited by immersion in a $0.3 \%(\mathrm{v} / \mathrm{v})$ hydrogen peroxide $\left(\mathrm{H}_{2} \mathrm{O}_{2}\right)$ in methanol bath for $15 \mathrm{~min}$. After washing, nonspecific binding was blocked with $5 \%(\mathrm{~m} / \mathrm{v})$ bovine serum albumin in PBS. The cells were incubated with diluted primary antibody Anti-SSEA4 antibody (15 $\mu \mathrm{g} / \mathrm{ml}$; Abcam), Anti-TRA-1-60 (R) antibody (1:100; Abcam), Anti-OCT4 antibody $(5 \mu \mathrm{g} / \mathrm{ml}$; Abcam), Anti-NANOG antibody (1 : 1000; Abcam), Anti-SOX2 antibody (1 $\mu \mathrm{g} / \mathrm{ml}$; Abcam), Anti-FABP4(1:1000; Abcam), Anti-PPAR gamma (5 $\mu \mathrm{g} / \mathrm{ml} ;$ Abcam),
Anti-RUNX2 (1:500; Abcam), Anti-Osteocalcin (10 $\mu \mathrm{g} / \mathrm{ml} ;$ Abcam), Anti-human AFP (1:200; Abcam), Anti-human CK18 (2 mg/ml, 1:400; Abcam), Anti-human CK19 (1:400; Abcam). According to the manufacturer's instructions, detection of primary antibody was performed using horseradish peroxidase-conjugated secondary antibodies (1 mg/ml, $1: 1000 ;$ Abcam). Peroxidase activity was revealed by a 3- to 5-min exposure to diaminobenzidine tetrahydrochloride solution (DAB kit, Vector Labs, Burlingame, CA, USA). The fixed cells were then washed, counterstained with hematoxylin for $1 \mathrm{~min}$, mounted and observed under an inverted phase-contrast microscope (ECLIPAS TS100, Nikon, Tokyo, Japan).

Periodic acid-Schiff staining. The medium was taken out from culture plates and cells were washed with PBS three times. Then, the cells were fixed using $4 \%$ paraformaldehyde (MultiSciences Biotech) for $30 \mathrm{~min}$. After being oxidized in periodic acid (Sigma) for $10 \mathrm{~min}$ and washed three times with PBS, cells were treated with Schiff's reagent (Sigma) for $15 \mathrm{~min}$. Afterwards, cells were rinsed in PBS for $10 \mathrm{~min}$ and counterstained with hematoxylin (Sigma). The staining results were observed under an inverted phase-contrast microscope (ECLIPAS TS100, Nikon).

Uptake and secretion of indocyanine green (ICG). Hepatogenesis differentiation medium was replaced with L-DMEM medium containing $1 \mathrm{mg} / \mathrm{ml}$ ICG (Sigma). After incubation at $37^{\circ} \mathrm{C}$ for $15 \mathrm{~min}$, the cells were rinsed three times with PBS and ICG uptake was measured using an inverted microscope. Dishes were refilled with general PDMSCs medium for $6 \mathrm{~h}$ and color changes were examined.

Gene expression profiling of adipogenic differentiated and dedifferentiated cells. After RNA extraction, all quantification and microarray experiments were performed at the Shanghai Biotechnology Corporation using Affymetrix PrimeView human gene expression (Affymetrix, Santa Clara, CA, USA). RNA integrity was analyzed using an Agilent Bioanalyzer 2100 (Agilent Technologies, Santa Clara, CA, USA). Qualified total RNA was further purified using an RNeasy micro kit (Qiagen, Hilden, Germany) and an RNase-Free DNase kit (QIAGEN). The RNA purity and concentration were determined using a Nanodrop 2000 (Nanodrop Products, Wilmington, DE, USA). Total RNA was amplified, labeled and purified using the GeneChip 3'IVT Express Kit (Affymetrix) following the manufacturer's instructions to obtain biotin-labeled cRNA. Array hybridization and washes were performed using the GeneChip Hybridization, Wash and Stain Kit (Affymetrix) in the Hybridization Oven 645 (Affymetrix) and Fluidics Station 450 (Affymetrix) following the manufacturer's instructions. Slides were scanned by the GeneChip Scanner 3000 (Affymetrix) and Command Console Software 4.0 (Affymetrix) with default settings. The scanned images were first assessed by visual inspection, and then analyzed to generate raw data files that were saved as CEL files using the default settings of GCOS 1.4. The raw data were normalized using an RMA algorithm in the Gene Spring Software 11.0 (Agilent Technologies). Expression profiling was performed for nine samples $(n=3$ donors) subdivided into three groups: $3 \times$ (PDMSCs), $3 \times$ (AL cells) and $3 \times$ (DePDMSCs P3). The microarray data sets have been submitted to the Gene Expression Omnibus (GEO) database and are accessible via the GEO ID: GSE73964.

Data normalization, selection criteria and analysis strategy. First, we were interested in genes whose expression was significantly up- or downregulated during the course of adipogenic differentiation. Thus, in the first step, each of the three AL cell GeneChips were compared with each of the three PDMSC GeneChips for comparative gene expression analysis. Genes were selected as differentially expressed on the basis of specific change call and fold change (FC) criteria. Changes in the $P$-value $<0.01$ and the FC limit $>2$ or $<-2$ were calculated for the mean $\mathrm{FC}$ of three comparisons to allow the selection of genes that were differentially expressed during adipogenesis. Next, we compared $\mathrm{AL}$ cell gene expression values with the corresponding values from DePDMSCs. The differentially expressed genes were selected according to the same criteria mentioned above. Third, we compared PDMSC gene expression values with the corresponding values from DePDMSCs. All differentially expressed genes were uploaded to the Database for Annotation, Visualization and Integrated Discovery (DAVID) 6.7 and analyzed according to the default set of statistical parameters. ${ }^{53}$ DAVID and the Kyoto Encyclopedia of Genes and Genomes (KEGG) were used for the evaluation and statistical analysis of genes. ${ }^{54}$ 
Statistical analyses. The change was calculated using the comparative cycles to threshold $(\mathrm{Ct})$ method. The calculation of gene expression was made as follows by comparing FABP4 expression of AL cells to PDMSCs: FABP4 (AL cells) $=2^{-\triangle \triangle \mathrm{Ct} ;} \triangle \triangle \mathrm{Ct}=[\mathrm{Ct}(\mathrm{FABP} 4)-\mathrm{Ct}(\beta$-actin)] AL cells $-[\mathrm{Ct}$ (FABP4) $-\mathrm{Ct}(\beta$-actin)] PDMSCs. All the experiments were conducted in triplicate, and the results are expressed as the mean \pm S.D. One-way ANOVA for three group comparison was performed by using SPSS 16.0 (SPSS, Chicago, IL, USA). $P<0.05$ was considered significant. The confidence interval was $95 \%$.

\section{Conflict of Interest}

The authors declare no conflict of interest.

Acknowledgements. This work was supported by the National Natural Science Foundation of China (No. 81471794), Chinese High Tech Research \& Development (863) Program (No. SS2013AA020102) and the National Science and Technology Major Project (No. 2012ZX10002004).

1. Hao J, Zhang Y, Jing D, Shen Y, Tang G, Huang S et al. Mechanobiology of mesenchymal stem cells: perspective into mechanical induction of MSC fate. Acta Biomater 2015; 20: 1-9.

2. Makhoul G, Chiu RC, Cecere R. Placental mesenchymal stem cells: a unique source for cellular cardiomyoplasty. Ann Thorac Surg 2013; 95: 1827-1833.

3. Walsh K, Perlman H. Cell cycle exit upon myogenic differentiation. Curr Opin Genet Dev 1997; 7: 597-602.

4. Zhang Y, Li TS, Lee ST, Wawrowsky KA, Cheng K, Galang G et al. Dedifferentiation and proliferation of mammalian cardiomyocytes. PLoS One 2010; 5: e12559.

5. Roobrouck VD, Clavel C, Jacobs SA, Ulloa-Montoya F, Crippa S, Sohni A et al. Differentiation potential of human postnatal mesenchymal stem cells, mesoangioblasts, and multipotent adult progenitor cells reflected in their transcriptome and partially influenced by the culture conditions. Stem Cells 2011; 29: 871-882.

6. Conrad S, Renninger M, Hennenlotter J, Wiesner T, Just L, Bonin M et al. Generation of pluripotent stem cells from adult human testis. Nature 2008; 456: 344-349.

7. Moschidou D, Mukherjee S, Blundell MP, Drews K, Jones GN, Abdulrazzak H et al. Valproic acid confers functional pluripotency to human amniotic fluid stem cells in a transgene-free approach. Mol Ther 2012; 20: 1953-1967.

8. Odelberg SJ, Kollhoff A, Keating MT. Dedifferentiation of mammalian myotubes induced by msx1. Cell 2000; 103: 1099-1109.

9. David L, Polo JM. Phases of reprogramming. Stem Cell Res 2014; 12: 754-761.

10. Park SJ, Yeo HC, Kang NY, Kim H, Lin J, Ha HH et al. Mechanistic elements and critical factors of cellular reprogramming revealed by stepwise global gene expression analyses. Stem Cell Res 2014; 12: 730-741

11. Redmer T, Diecke S, Grigoryan T, Quiroga-Negreira A, Birchmeier W, Besser D. E-cadherin is crucial for embryonic stem cell pluripotency and can replace OCT4 during somatic cell reprogramming. EMBO Rep 2011; 12: 720-726.

12. Song $L$, Webb NE, Song $Y$, Tuan RS. Identification and functional analysis of candidate genes regulating mesenchymal stem cell self-renewal and multipotency. Stem Cells 2006; 24: $1707-1718$.

13. Woodbury D, Reynolds K, Black IB. Adult bone marrow stromal stem cells express germline, ectodermal, endodermal, and mesodermal genes prior to neurogenesis. J Neurosci Res 2002; 69: 908-917.

14. Li TY, Shu C, Wong CH, Lo PS, Zhu H, Lau MC et al. Plasticity of rat bone marrow-derived 5-hydroxytryptamine-sensitive neurons: dedifferentiation and redifferentiation. Cell Biol Int 2004; 28: 801-807.

15. Ullah M, Stich S, Notter M, Eucker J, Sittinger M, Ringe J. Transdifferentiation of mesenchymal stem cells-derived adipogenic-differentiated cells into osteogenic- or chondrogenic-differentiated cells proceeds via dedifferentiation and have a correlation with cell cycle arresting and driving genes. Differentiation 2013; 85: 78-90.

16. Liu $Y$, Jiang $X$, Zhang $X$, Chen $R$, Sun $T$, Fok $K L$ et al. Dedifferentiation-reprogrammed mesenchymal stem cells with improved therapeutic potential. Stem Cells 2011; 29: 2077-2089.

17. Chen $\mathrm{CT}$, Hsu SH, Wei YH. Mitochondrial bioenergetic function and metabolic plasticity in stem cell differentiation and cellular reprogramming. Biochim Biophys Acta 2012; 1820: $571-576$.

18. Xu X, Duan S, Yi F, Ocampo A, Liu GH, Izpisua Belmonte JC. Mitochondrial regulation in pluripotent stem cells. Cell Metab 2013; 18: 325-332.

19. Cho YM, Kwon S, Pak YK, Seol HW, Choi YM, Park do J et al. Dynamic changes in mitochondrial biogenesis and antioxidant enzymes during the spontaneous differentiation of human embryonic stem cells. Biochem Biophys Res Commun 2006; 348 : 1472-1478.

20. Lonergan T, Bavister B, Brenner C. Mitochondria in stem cells. Mitochondrion 2007; 7: 289-296.
21. Benard G, Bellance N, Jose C, Melser S, Nouette-Gaulain K, Rossignol R. Multi-site control and regulation of mitochondrial energy production. Biochim Biophys Acta 2010; 1797: 698-709.

22. Gibson NJ. Cell adhesion molecules in context: CAM function depends on the neighborhood. Cell Adh Migr 2011; 5: 48-51.

23. Sharma NS, Nagrath D, Yarmush ML. Metabolic profiling based quantitative evaluation of hepatocellular metabolism in presence of adipocyte derived extracellular matrix. PLOS One 2011; 6: e20137.

24. Cao H, Yang J, Yu J, Pan Q, Li J, Zhou P et al. Therapeutic potential of transplanted placental mesenchymal stem cells in treating Chinese miniature pigs with acute liver failure. BMC Med 2012; 10: 56.

25. Moiseeva EP, Straatman KR, Leyland ML, Bradding P. CADM1 controls actin cytoskeleton assembly and regulates extracellular matrix adhesion in human mast cells. PLoS One 2014; 9: e85980.

26. Ladewig J, Koch P, Brustle O. Leveling Waddington: the emergence of direct programming and the loss of cell fate hierarchies. Nat Rev Mol Cell Biol 2013; 14: 225-236.

27. Jopling C, Boue S, Izpisua Belmonte JC. Dedifferentiation, transdifferentiation and reprogramming: three routes to regeneration. Nat Rev Mol Cell Biol 2011; 12: 79-89.

28. Jumabay M, Abdmaulen R, Ly A, Cubberly MR, Shahmirian LJ, Heydarkhan-Hagvall S et al. Pluripotent stem cells derived from mouse and human white mature adipocytes. Stem Cells Transl Med 2014; 3: 161-171.

29. Asada S, Kuroda M, Aoyagi Y, Fukaya Y, Tanaka S, Konno S et al. Ceiling culture-derived proliferative adipocytes retain high adipogenic potential suitable for use as a vehicle for gene transduction therapy. Am J Physiol Cell Physiol 2011; 301: C181-C185.

30. Zagoura DS, Trohatou O, Bitsika V, Makridakis M, Pappa KI, Vlahou A et al. AF-MSCs fate can be regulated by culture conditions. Cell Death Dis 2013; 4: e571.

31. Tanabe S, Sato Y, Suzuki T, Suzuki K, Nagao T, Yamaguchi T. Gene expression profiling of human mesenchymal stem cells for identification of novel markers in early- and late-stage cell culture. J Biochem 2008; 144: 399-408.

32. Khvorostov I, Zhang J, Teitell M. Probing for mitochondrial complex activity in human embryonic stem cells. J Vis Exp 2008 Jun 17;(16). pii: 724. doi:10.3791/724.

33. Palomaki S, Pietila M, Laitinen S, Pesala J, Sormunen R, Lehenkari $P$ et al. HIF-1alpha is upregulated in human mesenchymal stem cells. Stem Cells 2013; 31: 1902-1909.

34. Pietila M, Lehtonen S, Narhi M, Hassinen IE, Leskela HV, Aranko K et al. Mitochondrial function determines the viability and osteogenic potency of human mesenchymal stem cells. Tissue Eng Part C Methods 2010; 16: 435-445.

35. Pietila M, Palomaki S, Lehtonen S, Ritamo I, Valmu L, Nystedt J et al. Mitochondrial function and energy metabolism in umbilical cord blood- and bone marrow-derived mesenchymal stem cells. Stem Cells Dev 2012; 21: 575-588.

36. Hofmann AD, Beyer M, Krause-Buchholz U, Wobus M, Bornhauser M, Rodel G. OXPHOS supercomplexes as a hallmark of the mitochondrial phenotype of adipogenic differentiated human MSCs. PLoS One 2012; 7: e35160.

37. Zhang Y, Marsboom G, Toth PT, Rehman J. Mitochondrial respiration regulates 6pcadipogenic differentiation of human mesenchymal stem cells. PLOS One 2013; 8: e77077.

38. Wanet A, Remacle N, Najar M, Sokal E, Arnould T, Najimi M et al. Mitochondrial remodeling in hepatic differentiation and dedifferentiation. Int J Biochem Cell Biol 2014; 54: 174-185.

39. Chen CT, Shih YR, Kuo TK, Lee OK, Wei YH. Coordinated changes of mitochondrial biogenesis and antioxidant enzymes during osteogenic differentiation of human mesenchymal stem cells. Stem Cells 2008; 26: 960-968.

40. Vega-Naredo I, Loureiro R, Mesquita KA, Barbosa IA, Tavares LC, Branco AF et al. Mitochondrial metabolism directs stemness and differentiation in P19 embryonal carcinoma stem cells. Cell Death Differ 2014; 21: 1560-1574.

41. Chung S, Arrell DK, Faustino RS, Terzic A, Dzeja PP. Glycolytic network restructuring integral to the energetics of embryonic stem cell cardiac differentiation. $J$ Mol Cell Cardiol 2010; 48: 725-734.

42. Prigione A, Fauler B, Lurz R, Lehrach $\mathrm{H}$, Adjaye J. The senescence-related mitochondrial/ oxidative stress pathway is repressed in human induced pluripotent stem cells. Stem Cells 2010; 28: 721-733.

43. Suhr ST, Chang EA, Tjong J, Alcasid N, Perkins GA, Goissis MD et al. Mitochondrial rejuvenation after induced pluripotency. PLOS One 2010; 5: e14095.

44. Ronchi JA, Figueira TR, Ravagnani FG, Oliveira HC, Vercesi AE, Castilho RF. A spontaneous mutation in the nicotinamide nucleotide transhydrogenase gene of C57BL/6J mice results in mitochondrial redox abnormalities. Free Radic Biol Med 2013; 63: 446-456.

45. Weinberg-Shukron A, Abu-Libdeh A, Zhadeh F, Carmel L, Kogot-Levin A, Kamal L et al. Combined mineralocorticoid and glucocorticoid deficiency is caused by a novel founder nicotinamide nucleotide transhydrogenase mutation that alters mitochondrial morphology and increases oxidative stress. J Med Genet 2015; 52: 636-641.

46. Huang PI, Chen YC, Chen LH, Juan CC, Ku HH, Wang ST et al. PGC-1alpha mediates differentiation of mesenchymal stem cells to brown adipose cells. $J$ Atheroscler Thromb 2011; 18: 966-980.

47. Tormos KV, Anso E, Hamanaka RB, Eisenbart J, Joseph J, Kalyanaraman B et al. Mitochondrial complex III ROS regulate adipocyte differentiation. Cell Metab 2011; 14: 537-544. 
48. Berry N, Gursel DB, Boockvar JA. Direct conversion of human fibroblasts to functional neurons in one step. Neurosurgery 2011; 69: N18.

49. Szabo E, Rampalli S, Risueno RM, Schnerch A, Mitchell R, Fiebig-Comyn A et al. Direct conversion of human fibroblasts to multilineage blood progenitors. Nature 2010; 468: 521-526.

50. Mentink A, Hulsman M, Groen N, Licht R, Dechering KJ, van der Stok J et al. Predicting the therapeutic efficacy of MSC in bone tissue engineering using the molecular marker CADM1. Biomaterials 2013; 34: 4592-4601.

51. Ullah $M$, Sittinger $M$, Ringe J. Extracellular matrix of adipogenically differentiated mesenchymal stem cells reveals a network of collagen filaments, mostly interwoven by hexagonal structural units. Matrix Biol 2013; 32: 452-465.

52. Mariman EC, Wang P. Adipocyte extracellular matrix composition, dynamics and role in obesity. Cell Mol Life Sci 2010; 67: 1277-1292.

53. Huang da W, Sherman BT, Lempicki RA. Systematic and integrative analysis of large gene lists using DAVID bioinformatics resources. Nat Protoc 2009; 4: 44-57.

\section{Zhang JD, Wiemann S. KEGGgraph: a graph approach to KEGG PATHWAY in R and bioconductor. Bioinformatics 2009; 25: 1470-1471.}

cc) (i) Cell Death and Disease is an open-access journal published by Nature Publishing Group. This work is licensed under a Creative Commons Attribution 4.0 International License. The images or other third party material in this article are included in the article's Creative Commons license, unless indicated otherwise in the credit line; if the material is not included under the Creative Commons license, users will need to obtain permission from the license holder to reproduce the material. To view a copy of this license, visit http://creativecommons.org/licenses/by/4.0/

Supplementary Information accompanies this paper on Cell Death and Disease website (http://www.nature.com/cddis) 